\title{
PREPRINT
}

\section{The reconstruction of a time-dependent source from a surface measurement for full Maxwell's equations by means of the potential field method}

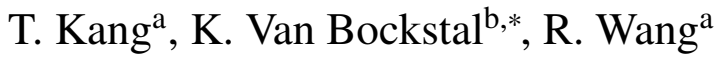 \\ ${ }^{a}$ Department of Applied Mathematics, School of Sciences, Communication University of China, \\ ${ }^{b}$ Department of Mathematical Analysis, research group of Numerical Analysis and Mathematical Modeling $\left(\mathrm{NaM}^{2}\right)$, \\ Ghent University, Galglaan 2 - S22, Gent 9000, Belgium
}

\begin{abstract}
This paper is devoted to the study of an inverse source problem governed by full Maxwell's equations by means of the potential field method (the $\boldsymbol{A}-\phi$ method). The source term is assumed to be separable in time and space, in which the unknown part is solely time-dependent and is recovered from a surface measurement. We prove that the solution to the inverse problem based on the $\boldsymbol{A}$ - $\phi$ formulation is existing and unique. We suggest a constructive scheme for approximating the solution and discuss its convergence. Finally, a few examples are presented to verify the theoretical results.
\end{abstract}

(C) 2017 Author's personal copy

Keywords: Maxwell's equations, inverse source problem, $\boldsymbol{A}-\phi$ method, reconstruction, time discretization 2000 MSC: 35Q60, 65N30, 65M60

\section{Introduction}

In this contribution, an inverse source problem governed by the full Maxwell's equations is studied by means of the potential field method (the $\boldsymbol{A}-\phi$ method). A bounded, simply-connected and convex polyhedron $\Omega \subset \mathbb{R}^{3}$ with connected boundary $\partial \Omega$ is considered. The outer normal vector associated with the boundary is denoted by $\boldsymbol{n}$. The current density $\boldsymbol{J}$ satisfies Ohm's law such that $\boldsymbol{J}=\sigma \boldsymbol{E}+\boldsymbol{J}_{a}$, where the vector function $\boldsymbol{J}_{a}$ describes possible current sources in $\Omega$. Electromagnetic fields are described by the full linear Maxwell's equations, i.e.

$$
\begin{cases}\partial_{t} \boldsymbol{D}(\boldsymbol{x}, t)-(\nabla \times \boldsymbol{H})(\boldsymbol{x}, t)=-\boldsymbol{J}, & (\boldsymbol{x}, t) \in \Omega \times(0, T] \\ \partial_{t} \boldsymbol{B}(\boldsymbol{x}, t)+(\nabla \times \boldsymbol{E})(\boldsymbol{x}, t)=\mathbf{0}, & (\boldsymbol{x}, t) \in \Omega \times(0, T]\end{cases}
$$

or in terms of $\boldsymbol{E}$ and $\boldsymbol{H}$ as

$$
\begin{cases}\varepsilon(\boldsymbol{x}) \partial_{t} \boldsymbol{E}(\boldsymbol{x}, t)-(\nabla \times \boldsymbol{H})(\boldsymbol{x}, t)=-\left(\sigma(\boldsymbol{x}) \boldsymbol{E}(\boldsymbol{x}, t)+\boldsymbol{J}_{a}(\boldsymbol{x}, t)\right), & (\boldsymbol{x}, t) \in \Omega \times(0, T] \\ \mu(\boldsymbol{x}) \partial_{t} \boldsymbol{H}(\boldsymbol{x}, t)+(\nabla \times \boldsymbol{E})(\boldsymbol{x}, t)=\mathbf{0}, & (\boldsymbol{x}, t) \in \Omega \times(0, T]\end{cases}
$$

where $\boldsymbol{D}, \boldsymbol{E}, \boldsymbol{B}$ and $\boldsymbol{H}$ are the electric displacement field, electric field, magnetic induction and the magnetic field, $\sigma, \varepsilon$ and $\mu$ denote the electric conductivity, the electric permittivity and the magnetic permeability of the medium, respectively.

\footnotetext{
${ }^{*}$ Corresponding author

Email addresses: kangtong@cuc.edu.cn (T. Kang), karel.vanbockstal@ugent.be (K. Van Bockstal), ranwang@cuc.edu.cn (R. Wang)

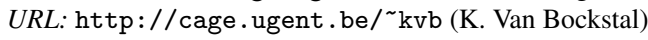


The source term $\boldsymbol{J}_{a}$ is assumed to be separable, i.e.

$$
\boldsymbol{J}_{a}(\boldsymbol{x}, t)=h(t) \boldsymbol{f}(\boldsymbol{x})
$$

where $\boldsymbol{f}(\boldsymbol{x})$ is given but $h(t)$ is unknown. The measurement used to recover $h(t)$ is specified later. It is assumed that the field $f$ obeys

$$
\boldsymbol{f} \times \boldsymbol{n}=\mathbf{0} \quad \text { on } \partial \mathbf{\Omega} .
$$

The whole domain $\Omega$ can be divided into finite subdomains. In each subdomain, the electric conductivity $\sigma$, the magnetic permeability $\mu$ and the electric permittivity $\varepsilon$ of the medium are positive constants. However, for simplicity, it is assumed that $\Omega$ consists of only two subdomains, namely a conducting subdomain $\Omega_{1}=: \Omega_{c}$ and a nonconducting subdomain $\Omega_{e}:=\Omega_{2}=\Omega \backslash \bar{\Omega}_{1}$. The electric conductivity $\sigma$ is thus given by

$$
\sigma= \begin{cases}\sigma_{c} & \text { in } \bar{\Omega}_{c}, \\ 0 & \text { in } \Omega \backslash \bar{\Omega}_{c},\end{cases}
$$

with $0<\sigma_{c}<\infty$. Further, it is assumed that

$$
0<\mu_{\min } \leq \mu \leq \mu_{\max } \quad \text { and } \quad 0<\varepsilon_{\min } \leq \varepsilon \leq \varepsilon_{\max }
$$

with $\mu_{\min }, \mu_{\max }, \varepsilon_{\min }$ and $\varepsilon_{\max }$ all strict positive constants. The interface between both domains is denoted by $\Gamma=\bar{\Omega}_{1} \cap \bar{\Omega}_{2}$.

Following the setting formulated above, the system (1.1) is equipped with the following boundary and initial conditions

$$
\begin{cases}\boldsymbol{n} \times \boldsymbol{E}(\boldsymbol{x}, t)=\mathbf{0}, & (\boldsymbol{x}, t) \in \partial \Omega \times(0, T], \\ \boldsymbol{E}(\boldsymbol{x}, 0)=\boldsymbol{E}_{0}, & \boldsymbol{x} \in \Omega, \\ {\left[\boldsymbol{n}_{12} \cdot\left(\varepsilon \partial_{t} \boldsymbol{E}+\sigma \boldsymbol{E}+h \boldsymbol{f}\right)\right]=\left[\boldsymbol{n}_{12} \cdot(\nabla \times \boldsymbol{H})\right]=\mathbf{0},} & \text { on } \Gamma \times(0, T], \\ \mu(\boldsymbol{x}) \boldsymbol{H}(\boldsymbol{x}, t) \cdot \boldsymbol{n}=\mathbf{0}, & (\boldsymbol{x}, t) \in \partial \Omega \times(0, T], \\ \boldsymbol{H}(\boldsymbol{x}, 0)=\boldsymbol{H}_{0}, & \boldsymbol{x} \in \Omega, \\ {\left[\boldsymbol{n}_{12} \times \boldsymbol{H}\right]=\mathbf{0},} & \text { on } \Gamma \times(0, T],\end{cases}
$$

with

$$
\left(\nabla \cdot \boldsymbol{B}_{0}\right)(\boldsymbol{x})=\left(\nabla \cdot\left(\mu \boldsymbol{H}_{0}\right)\right)(\boldsymbol{x})=0, \quad \boldsymbol{x} \in \Omega,
$$

to ensure that the magnetic induction is divergence free. The unit normal vector $\boldsymbol{n}_{12}$ points from $\Omega_{1}$ to $\Omega_{2}$, thus the jump is defined as follows

$$
\left[\boldsymbol{n}_{12} \times \boldsymbol{u}\right]=\boldsymbol{n} \times\left(\boldsymbol{u}_{2}-\boldsymbol{u}_{1}\right), \quad\left[\boldsymbol{n}_{12} \cdot \boldsymbol{u}\right]=\boldsymbol{n} \cdot\left(\boldsymbol{u}_{2}-\boldsymbol{u}_{1}\right) .
$$

Usually, the Maxwell system is transformed to the $\boldsymbol{E}$ or $\boldsymbol{H}$ equation and the resulting equation is solved by using edge finite element methods. Besides, it can also be changed into potential formulations by means of a decomposition of the field $\boldsymbol{E}$ or $\boldsymbol{H}$ (the so-called $\boldsymbol{A}-\phi$ or $\boldsymbol{T}-\psi$ method). Then, nodal finite elements are used to solve the equation numerically, cf. $[1,3,7,8,13,22,23]$. There are several advantages for the potential field method. For example, it can deal with the possible discontinuity between different mediums very well and has good numerical accuracy. The method avoids spurious solutions by adding a penalty function term in the dominant equation. Moreover, it also has attractive features including natural coupling to moment and boundary element methods, and global energy conservation.

There are many works on inverse problems for electromagnetic fields based on the $\boldsymbol{E}$ or $\boldsymbol{H}$ equation, e.g. [5, 6, 14, $15,18,21]$. But research upon the potential field method has been rarely found. The purpose of this paper is to study the inverse source problem specified above by employing the $\boldsymbol{A}-\phi$ method. To transform the formulation (1.1) to the $\boldsymbol{A}-\boldsymbol{\phi}$ method, first a potential vector $\boldsymbol{A}$ is introduced, defined by (see [4, Theorem 2.2])

$$
\boldsymbol{H}=\frac{1}{\mu} \nabla \times \boldsymbol{A} \quad \text { with } \quad \nabla \cdot \boldsymbol{A}=0 \text { in } \Omega \quad \text { and } \quad \boldsymbol{n} \times \boldsymbol{A}=\mathbf{0} \text { on } \partial \Omega,
$$

and thus (using the second equation in (1.1))

$$
\boldsymbol{E}=-\partial_{t} \boldsymbol{A}-\nabla \partial_{t} \phi
$$

where the function $\phi$ is determined up to an additive constant. To ensure the uniqueness of $\phi$, it is assumed that $\phi=0$ on $\partial \Omega$. Now, the first equation of (1.1) can be rewritten as

$$
\varepsilon \partial_{t t}(\boldsymbol{A}+\nabla \phi)+\sigma \partial_{t}(\boldsymbol{A}+\nabla \phi)+\nabla \times\left(\frac{1}{\mu} \nabla \times \boldsymbol{A}\right)=h \boldsymbol{f},
$$


Moreover, the divergence of both sides of (1.3) has been taken to get that

$$
\nabla \cdot\left(\varepsilon \partial_{t t}(\boldsymbol{A}+\nabla \phi)+\sigma \partial_{t}(\boldsymbol{A}+\nabla \phi)\right)=h \nabla \cdot \boldsymbol{f}
$$

12

A penalty function term $-\nabla\left(\frac{1}{\mu} \nabla \cdot \boldsymbol{A}\right)$ is added into the dominant $\boldsymbol{A}-\phi$ equation to ensure that the vector potential $\boldsymbol{A}$ is divergence-free (see, e.g. [23]). The $\boldsymbol{A}$ - $\boldsymbol{\phi}$ formulation reads as follows:

The dominant equations in the $\boldsymbol{A}-\phi$ formulation are given by

$$
\begin{cases}\varepsilon \partial_{t t}(\boldsymbol{A}+\nabla \phi)+\sigma \partial_{t}(\boldsymbol{A}+\nabla \phi)+\nabla \times\left(\frac{1}{\mu} \nabla \times \boldsymbol{A}\right)-\nabla\left(\frac{1}{\mu} \nabla \cdot \boldsymbol{A}\right)=h \boldsymbol{f}, & \text { in } \Omega \times(0, T], \\ \nabla \cdot\left(\varepsilon \partial_{t t}(\boldsymbol{A}+\nabla \phi)+\sigma \partial_{t}(\boldsymbol{A}+\nabla \phi)\right)=h \nabla \cdot \boldsymbol{f}, & \text { in } \Omega \times(0, T], \\ \boldsymbol{A}(\boldsymbol{x}, 0)=\boldsymbol{A}_{0}, \partial_{t} \boldsymbol{A}(\boldsymbol{x}, 0)=\boldsymbol{A}_{0}^{\prime}, & \boldsymbol{x} \in \Omega, \\ \phi(\boldsymbol{x}, 0)=\phi_{0}, \partial_{t} \phi(\boldsymbol{x}, 0)=\phi_{0}^{\prime}, & \boldsymbol{x} \in \Omega,\end{cases}
$$

the boundary conditions on $\partial \Omega$ are given by

$$
\begin{cases}\boldsymbol{n} \times \boldsymbol{A}(\boldsymbol{x}, t)=\mathbf{0}, & (\boldsymbol{x}, t) \in \partial \Omega \times(0, T], \\ \nabla \cdot \boldsymbol{A}(\boldsymbol{x}, t)=0, & (\boldsymbol{x}, t) \in \partial \Omega \times(0, T], \\ \phi(\boldsymbol{x}, t)=0, & (\boldsymbol{x}, t) \in \partial \Omega \times(0, T],\end{cases}
$$

and the interface conditions on $\Gamma$ are given by

$$
\begin{aligned}
& {[\phi]=0, \quad \text { on } \Gamma \times(0, T],} \\
& {[\boldsymbol{A}]=\mathbf{0}, \quad \text { on } \Gamma \times(0, T],} \\
& {\left[\boldsymbol{n}_{12} \cdot\left(\varepsilon \frac{\partial^{2}}{\partial t^{2}}(\boldsymbol{A}+\nabla \phi)+\sigma \frac{\partial}{\partial t}(\boldsymbol{A}+\nabla \phi)-h \boldsymbol{f}\right)\right]=\left[\boldsymbol{n}_{12} \cdot\left(\nabla \times\left(\frac{1}{\mu} \nabla \times \boldsymbol{A}\right)-\nabla\left(\frac{1}{\mu} \nabla \cdot \boldsymbol{A}\right)\right)\right]=0, \quad \text { on } \Gamma \times(0, T],} \\
& {\left[\boldsymbol{n}_{12} \times\left(\frac{1}{\mu} \nabla \times \boldsymbol{A}\right)\right]=\mathbf{0}, \quad \text { on } \Gamma \times(0, T],} \\
& {\left[\frac{1}{\mu} \nabla \cdot A\right]=0, \quad \text { on } \Gamma \times(0, T],}
\end{aligned}
$$

where the function $\boldsymbol{A}_{0}$ is derived from $\boldsymbol{H}_{0} \in \boldsymbol{L}^{2}(\Omega)$ by

$$
\nabla \times \boldsymbol{A}_{0}=\mu \boldsymbol{H}_{0} \quad \text { with } \quad \nabla \cdot \boldsymbol{A}_{0}=0 \text { in } \Omega,
$$

where the function $\boldsymbol{A}_{0}^{\prime}$ is calculated from $\boldsymbol{E}_{0} \in \boldsymbol{H}(\operatorname{curl}, \Omega) \cap \boldsymbol{H}(\operatorname{div}, \Omega)$ by

$$
\nabla \times \boldsymbol{A}_{0}^{\prime}=-\nabla \times \boldsymbol{E}_{0} \quad \text { with } \quad \nabla \cdot \boldsymbol{A}_{0}^{\prime}=0 \text { in } \Omega,
$$

where $\phi_{0}^{\prime}$ is found from

$$
-\Delta \phi_{0}^{\prime}=\nabla \cdot \boldsymbol{E}_{0} \quad \text { with } \quad \phi_{0}^{\prime}=0 \text { on } \partial \Omega
$$

and $\phi_{0}$ is defined by

$$
\phi_{0}=t_{1} \phi_{0}^{\prime}
$$
assumming that $\phi\left(-t_{1}\right)=0$ is prescribed for small $t_{1}>0$ (in computations usually $t_{1}$ is taken the time step).

The interface conditions (1.6)-(1.7) are natural conditions when using nodal finite elements, the interface condition (1.8) means the continuity of the current density in normal direction, the condition (1.9) reflects the continuity of magnetic field in tangential direction, and the condition (1.10) ensures that the vector potential field $\boldsymbol{A}$ is divergence-free on the whole domain.

The inverse source problem here is to find a triple $(\boldsymbol{A}(\boldsymbol{x}, t), \phi(\boldsymbol{x}, t), h(t))$ from which $\boldsymbol{E}$ and $\boldsymbol{H}$ can be derived. Usually, an integral overdetermination measurement is applied to this type of inverse problems for evolutionary problems, cf. $[10,16,17,18,20,27]$ and the references therein. Most of the existing literature shows that the additional integral measurement is taken over the whole domain (or over a subdomain). However, the way to obtain such a measurement is not always obvious from practical point of view. Note that if the unknown source depends on the space variable, one usual needs an additional space measurement (e.g. the solution at the final time), cf. [2, 9, 11, 19, 24]. This means that both kinds of inverse source problems need often totally different additional data. 
In this contribution, the missing data function $h(t)$ is recovered by means of the following normal component measurement along a $2 \mathrm{D}$-surface $\gamma \subset \bar{\Omega}_{c}$ :

$$
\int_{\gamma} \boldsymbol{E} \cdot \boldsymbol{n} d \gamma=-\int_{\gamma}\left(\partial_{t} \boldsymbol{A}+\nabla \partial_{t} \phi\right) \cdot \boldsymbol{n} d \gamma=m(t)
$$

2. Notations and time discretization

For convenience of presentation, we first give some notations that are used throughout this paper. Let $L^{2}(\Omega)$ be the usual Hilbert space of square integrable functions equipped with the inner product and norm:

$$
(u, v):=\int_{\Omega} u(\boldsymbol{x}) v(\boldsymbol{x}) d \boldsymbol{x} \text { and }\|u\|_{L^{2}(\Omega)}:=(u, u)^{1 / 2}
$$

Define $H^{m}(\Omega):=\left\{v \in L^{2}(\Omega): D^{\xi} v \in L^{2}(\Omega),|\xi| \leq m\right\}$, which is equipped with the following norm and semi-norm

$$
\|u\|_{H^{m}(\Omega)}:=\left(\sum_{|\xi| \leq m}\left\|D^{\xi} u\right\|_{L^{2}(\Omega)}^{2}\right)^{1 / 2} \text { and }|u|_{H^{m}(\Omega)}:=\left(\sum_{|\xi|=m}\left\|D^{\xi} u\right\|_{L^{2}(\Omega)}^{2}\right)^{1 / 2},
$$

where $\xi$ represents a non-negative triple index. $H_{0}^{1}(\Omega)$ is the subspace of $H^{1}(\Omega)$ whose functions have zero traces on $\partial \Omega$. We use boldface notation to represent vector-valued quantities such as $\boldsymbol{L}^{2}(\Omega):=\left(L^{2}(\Omega)\right)^{3}$. Let $\widehat{\boldsymbol{H}}_{0}^{1}(\Omega):=\{\boldsymbol{v} \in$ $\boldsymbol{H}^{1}(\Omega): \boldsymbol{v} \times \boldsymbol{n}=\mathbf{0}$ on $\left.\partial \Omega\right\}$ with $\|\boldsymbol{v}\|_{\widehat{\boldsymbol{H}}_{0}^{1}(\Omega)}:=\|\boldsymbol{v}\|_{\boldsymbol{H}^{1}(\Omega)}$ for $\boldsymbol{v} \in \widehat{\boldsymbol{H}}_{0}^{1}(\Omega)$. The space $\boldsymbol{V}:=\widehat{\boldsymbol{H}}_{0}^{1}(\Omega) \times H_{0}^{1}(\Omega)$ is equipped with the inner product

$$
((\boldsymbol{P}, \varphi),(\boldsymbol{Q}, \psi))_{V}:=(\boldsymbol{P}+\nabla \varphi, \boldsymbol{Q}+\nabla \psi)+(\nabla \times \boldsymbol{P}, \nabla \times \boldsymbol{Q})+(\nabla \cdot \boldsymbol{P}, \nabla \cdot \boldsymbol{Q})
$$

and the norm

$$
\|(\boldsymbol{Q}, \psi)\|_{V}:=\left(\|\boldsymbol{Q}+\nabla \psi\|_{\boldsymbol{L}^{2}(\Omega)}^{2}+\|\nabla \times \boldsymbol{Q}\|_{\boldsymbol{L}^{2}(\Omega)}^{2}+\|\nabla \cdot \boldsymbol{Q}\|_{L^{2}(\Omega)}^{2}\right)^{1 / 2}
$$

Next, we show the following lemmas borrowed from [8]. A direct application of these lemmas yields that the $V$-norm 40 is equivalent to the norm $\left(\|\cdot\|_{\boldsymbol{H}^{1}(\Omega)}^{2}+\|\nabla \cdot\|_{L^{2}(\Omega)}^{2}\right)^{\frac{1}{2}}$.

Lemma 2.1 (coercivity). Let $\Omega$ be a convex bounded polyhedron. Then there exists a constant $C>0$ such that

$$
C\|(\boldsymbol{Q}, \psi)\|_{V}^{2} \geq\|\boldsymbol{Q}\|_{\boldsymbol{H}^{1}(\Omega)}^{2}+\|\nabla \psi\|_{\boldsymbol{L}^{2}(\Omega)}^{2}
$$

${ }_{41}$ for all $(\boldsymbol{Q}, \psi) \in \boldsymbol{V}$.

Lemma 2.2 (continuity). Let $\Omega$ be a convex bounded polyhedron. There exists a constant $C>0$ such that

$$
((\boldsymbol{P}, \varphi),(\boldsymbol{Q}, \psi))_{V}^{2} \leq C\left(\|\boldsymbol{P}\|_{\boldsymbol{H}^{1}(\Omega)}^{2}+\|\nabla \varphi\|_{\boldsymbol{L}^{2}(\Omega)}^{2}\right)\left(\|\boldsymbol{Q}\|_{\boldsymbol{H}^{1}(\Omega)}^{2}+\|\nabla \psi\|_{\boldsymbol{L}^{2}(\Omega)}^{2}\right)
$$

42 for any $(\boldsymbol{P}, \varphi),(\boldsymbol{Q}, \psi) \in \boldsymbol{V}$. 
Now, we can define the weak solution of (1.4) in suitable spaces. First, applying the measurement operator to the first equation of (1.4), we can eliminate the unknown $h(t)$ as follows

$$
\begin{gathered}
h(t)=\frac{-\varepsilon_{c} m^{\prime}(t)-\sigma_{c} m(t)+\frac{1}{\mu_{c}} \int_{\gamma} \nabla \times \nabla \times \boldsymbol{A} \cdot \boldsymbol{n} d \gamma-\frac{1}{\mu_{c}} \int_{\gamma} \nabla(\nabla \cdot \boldsymbol{A}) \cdot \boldsymbol{n} d \gamma}{\int_{\gamma} \boldsymbol{f} \cdot \boldsymbol{n} d \gamma} \\
\text { if } \int_{\gamma} \boldsymbol{f} \cdot \boldsymbol{n} d \gamma \neq 0 .
\end{gathered}
$$

Next, we take the scalar product of the first equation in (1.4) with $\boldsymbol{Q} \in \widehat{\boldsymbol{H}}_{0}^{1}\left(\Omega_{c}\right)$ and the scalar product of the second equation in (1.4) with $\psi \in H_{0}^{1}\left(\Omega_{c}\right)$. Afterwards, we integrate the result over the domain $\Omega_{c}$ and we apply Green's theorems. Afterwards, we repeat the previous step for $\Omega_{e}$. Then, the resulting formulations are added together to obtain for all $(Q, \psi) \in \boldsymbol{V}$ that

$$
\begin{aligned}
&\left(\varepsilon \partial_{t t}(\boldsymbol{A}(t)+\nabla \phi(t)), \boldsymbol{Q}+\nabla \psi\right)+\sigma_{c}\left(\partial_{t}(\boldsymbol{A}(t)+\nabla \phi(t)), \boldsymbol{Q}+\nabla \psi\right)_{\Omega_{c}}+\left(\frac{1}{\mu} \nabla \times \boldsymbol{A}(t), \nabla \times \boldsymbol{Q}\right) \\
&+\left(\frac{1}{\mu} \nabla \cdot \boldsymbol{A}(t), \nabla \cdot \boldsymbol{Q}\right)-\left(\left[\boldsymbol{n}_{12} \times(\nabla \times \boldsymbol{A}(t))\right], \boldsymbol{Q}\right)_{\Gamma}+\left([\nabla \cdot \boldsymbol{A}(t)], \boldsymbol{Q} \cdot \boldsymbol{n}_{12}\right)_{\Gamma} \\
& \quad-\left(\left[h \boldsymbol{f} \cdot \boldsymbol{n}_{12}-\varepsilon \partial_{t t}(\boldsymbol{A}+\nabla \phi) \cdot \boldsymbol{n}_{12}-\sigma \partial_{t}(\boldsymbol{A}+\nabla \phi) \cdot \boldsymbol{n}_{12}\right], \psi\right)_{\Gamma}=(h(t) \boldsymbol{f}, \boldsymbol{Q}+\nabla \psi) .
\end{aligned}
$$

43 The interface integrals cancel out by the interface conditions (1.8)-(1.10) such that the following condition can be stated.

Definition 2.1. The potential field pair $(\boldsymbol{A}, \phi)$, satisfying

$$
(\boldsymbol{A}(t), \phi(t)) \in \boldsymbol{V},\left(\partial_{t} \boldsymbol{A}(t), \partial_{t} \phi(t)\right) \in \boldsymbol{L}^{2}\left(\Omega_{c}\right) \times H^{1}\left(\Omega_{c}\right),\left(\partial_{t t} \boldsymbol{A}(t), \partial_{t t} \phi(t)\right) \in \boldsymbol{L}^{2}(\Omega) \times H^{1}(\Omega)
$$

is the weak solution of (1.4) if the following identity is fulfilled for a.a. $t \in[0, T]$ :

$$
\left\{\begin{array}{cl}
\left(\varepsilon \partial_{t t}(\boldsymbol{A}(t)+\nabla \phi(t)), \boldsymbol{Q}+\nabla \psi\right)+\sigma_{c}\left(\partial_{t}(\boldsymbol{A}(t)+\nabla \phi(t)), \boldsymbol{Q}+\nabla \psi\right)_{\Omega_{c}} & \\
+\left(\frac{1}{\mu} \nabla \times \boldsymbol{A}(t), \nabla \times \boldsymbol{Q}\right)+\left(\frac{1}{\mu} \nabla \cdot \boldsymbol{A}(t), \nabla \cdot \boldsymbol{Q}\right)=(h(t) \boldsymbol{f}, \boldsymbol{Q}+\nabla \psi), & \forall(\boldsymbol{Q}, \psi) \in \boldsymbol{V}, \\
\boldsymbol{A}(\boldsymbol{x}, 0)=\boldsymbol{A}_{0}, \partial_{t} \boldsymbol{A}(\boldsymbol{x}, 0)=\boldsymbol{A}_{0}^{\prime}, \phi(\boldsymbol{x}, 0)=\phi_{0}, \partial_{t} \phi(\boldsymbol{x}, 0)=\phi_{0}^{\prime}, & \boldsymbol{x} \in \Omega .
\end{array}\right.
$$

Remark 2.1. The variational formulation of the first and second equation of (1.4) is a special case of the formulation (2.3). Derivations similar to (2.2) are not repeated in detail further in the paper. Throughout the paper, the sentence 'integrate over $\Omega$ ' means sometimes these underlying calculations.

Let $n$ be a positive integer and $\left\{t_{i}=i \tau: i=0, \cdots, n\right\}$ be a equidistant partition of $[0, T]$ with $\tau=T / n$. Set for any function $u$

$$
u_{i}=u_{i}(\boldsymbol{x}) \approx u\left(\boldsymbol{x}, t_{i}\right), \partial_{t} u\left(\boldsymbol{x}, t_{i}\right) \approx \delta u_{i}=\frac{u_{i}-u_{i-1}}{\tau}, \partial_{t t} u\left(\boldsymbol{x}, t_{i}\right) \approx \delta^{2} u_{i}=\frac{\delta u_{i}-\delta u_{i-1}}{\tau} .
$$

With these notations, we are allowed to consider the following time-discrete problem: Given $\left(\boldsymbol{A}_{0}, \phi_{0}\right)$ and $\left(\delta \boldsymbol{A}_{0}, \delta \phi_{0}\right)=$ $\left(\boldsymbol{A}_{0}^{\prime}, \phi_{0}^{\prime}\right)$, find $\left(\boldsymbol{A}_{i}, \phi_{i}\right) \in \boldsymbol{V}$ for $i=1, \cdots, n$ such that

$$
\begin{cases}\varepsilon \delta^{2}\left(\boldsymbol{A}_{i}+\nabla \phi_{i}\right)+\sigma \delta\left(\boldsymbol{A}_{i}+\nabla \phi_{i}\right)+\nabla \times\left(\frac{1}{\mu} \nabla \times \boldsymbol{A}_{i}\right)-\nabla\left(\frac{1}{\mu} \nabla \cdot \boldsymbol{A}_{i}\right)=h_{i} \boldsymbol{f} & \text { in } \Omega \\ \nabla \cdot\left(\varepsilon \delta^{2}\left(\boldsymbol{A}_{i}+\nabla \phi_{i}\right)+\sigma \delta\left(\boldsymbol{A}_{i}+\nabla \phi_{i}\right)\right)=h_{i} \nabla \cdot \boldsymbol{f} & \text { in } \Omega \\ \boldsymbol{n} \times \boldsymbol{A}_{i}=\mathbf{0}, \nabla \cdot \boldsymbol{A}_{i}=0, \phi_{i}=0 & \text { on } \partial \Omega\end{cases}
$$

with

$$
h_{i}=\frac{-\varepsilon_{c} m_{i}^{\prime}-\sigma_{c} m_{i}+\frac{1}{\mu_{c}} \int_{\gamma} \nabla \times \nabla \times \boldsymbol{A}_{i-1} \cdot \boldsymbol{n} d \gamma-\frac{1}{\mu_{c}} \int_{\gamma} \nabla\left(\nabla \cdot \boldsymbol{A}_{i-1}\right) \cdot \boldsymbol{n} d \gamma}{\int_{\gamma} \boldsymbol{f} \cdot \boldsymbol{n} d \gamma} .
$$

Here (2.4)-(2.5) is a linear decoupled scheme. For a given $i$, we first derive $h_{i}$ and then solve (2.4). Afterwards, we replace $i$ with $i+1$ and repeat this process.

Theorem 2.3. Suppose that $\boldsymbol{f} \in \widehat{\boldsymbol{H}}_{0}^{1}(\Omega),\left(\boldsymbol{A}_{0}, \phi_{0}\right) \in \boldsymbol{V},\left(\boldsymbol{A}_{0}^{\prime}, \phi_{0}^{\prime}\right) \in \boldsymbol{V}, \nabla \times \nabla \times \boldsymbol{A}_{0} \in \widehat{\boldsymbol{H}}_{0}^{1}(\Omega), \nabla \cdot \boldsymbol{A}_{0}=\nabla \cdot \boldsymbol{A}_{0}^{\prime}=0$ in $\Omega$, $m \in C^{1}([0, T])$ and $\int_{\gamma} \boldsymbol{f} \cdot \boldsymbol{n} d \gamma \neq 0$. Then, for any $i=1, \cdots, n$, there exists a unique weak solution $\left(\boldsymbol{A}_{i}, \phi_{i}, h_{i}\right) \in \boldsymbol{V} \times \mathbb{R}$ for (2.4) and (2.5). Moreover, we have that $h_{i} \in \mathbb{R},\left(\boldsymbol{A}_{i}, \phi_{i}\right) \in \boldsymbol{V}, \nabla \times\left(\frac{1}{\mu} \nabla \times \boldsymbol{A}_{i}\right) \in \widehat{\boldsymbol{H}}_{0}^{1}(\Omega),\left[\nabla \times\left(\frac{1}{\mu} \nabla \times \boldsymbol{A}_{i}\right) \times \boldsymbol{n}_{12}\right]=\mathbf{0}$ on $\Gamma$ 
Proof. For a known $\left(\boldsymbol{A}_{i-1}, \phi_{i-1}\right) \in \boldsymbol{V}$ and a known $\left(\delta \boldsymbol{A}_{i-1}, \delta \phi_{i-1}\right)$, satisfying $\nabla \times \nabla \times \boldsymbol{A}_{i-1} \in \widehat{\boldsymbol{H}}_{0}^{1}(\Omega)$ and $\nabla \cdot \boldsymbol{A}_{i-1}=0$, we can compute $h_{i}$ from (2.5). We successively deduce that

$$
\begin{aligned}
\left|h_{i}\right|^{2} \quad= & \left|\frac{-\varepsilon_{c} m_{i}^{\prime}-\sigma_{c} m_{i}+\frac{1}{\mu_{c}} \int_{\gamma} \nabla \times \nabla \times \boldsymbol{A}_{i-1} \cdot \boldsymbol{n} d \gamma}{\int_{\gamma} \boldsymbol{f} \cdot \boldsymbol{n} d \gamma}\right|^{2} \\
\leq & C\left(1+\left\|\nabla \times \nabla \times \boldsymbol{A}_{i-1}\right\|_{\mathbf{L}^{2}(\gamma)}^{2}\right) \\
\substack{\gamma \subset \partial G \\
\leq} & C\left(1+\left\|\nabla \times \nabla \times \boldsymbol{A}_{i-1}\right\|_{\boldsymbol{L}^{2}(\partial G)}^{2}\right) \\
\boldsymbol{H}^{1}(G) \hookrightarrow \boldsymbol{L}^{2}(\partial G) & C\left(1+\left\|\nabla \times \nabla \times \boldsymbol{A}_{i-1}\right\|_{\boldsymbol{H}^{1}(G)}^{2}\right) \\
\leq & \left(1+\left\|\nabla \times \nabla \times \boldsymbol{A}_{i-1}\right\|_{\boldsymbol{H}^{1}(\Omega)}^{G^{\prime} \subset \Omega}\right) \\
\leq & C(1+\| \\
\leq & C_{i} .
\end{aligned}
$$

The semi-discrete approximation to (2.4) reads: Given $h_{i},\left(\boldsymbol{A}_{0}, \phi_{0}\right)$ and $\left(\delta \boldsymbol{A}_{0}, \delta \phi_{0}\right)=\left(\boldsymbol{A}_{0}^{\prime}, \phi_{0}^{\prime}\right)$, find $\left(\boldsymbol{A}_{i}, \phi_{i}\right) \in \boldsymbol{V}$, $1 \leq i \leq n$, such that

$$
\begin{aligned}
\left(\varepsilon \delta^{2}\left(\boldsymbol{A}_{i}+\nabla \phi_{i}\right), \boldsymbol{Q}+\nabla \psi\right)+\sigma_{c}\left(\delta\left(\boldsymbol{A}_{i}+\nabla \phi_{i}\right), \boldsymbol{Q}+\nabla \psi\right)_{\Omega_{c}} & +\left(\frac{1}{\mu} \nabla \times \boldsymbol{A}_{i}, \nabla \times \boldsymbol{Q}\right) \\
& +\left(\frac{1}{\mu} \nabla \cdot \boldsymbol{A}_{i}, \nabla \cdot \boldsymbol{Q}\right)=\left(h_{i} \boldsymbol{f}, \boldsymbol{Q}+\nabla \psi\right), \quad \forall(\boldsymbol{Q}, \psi) \in \boldsymbol{V} .
\end{aligned}
$$
that

Taking the divergence to the both sides of the first equation of (2.4) and taking into account the second equation yield

$$
\nabla^{2}\left(\frac{1}{\mu} \nabla \cdot \boldsymbol{A}_{i}\right)=0 \quad \text { in } \Omega .
$$

Considering the boundary condition $\nabla \cdot \boldsymbol{A}_{i}=0$, we obtain that

$$
\nabla \cdot \boldsymbol{A}_{i}=0 \quad \text { a.e. in } \Omega .
$$

Therefore, we get that

$$
\begin{gathered}
\nabla \times\left(\frac{1}{\mu} \nabla \times \boldsymbol{A}_{i}\right)=h_{i} f-\varepsilon \delta^{2}\left(\boldsymbol{A}_{i}+\nabla \phi_{i}\right)-\sigma \delta\left(\boldsymbol{A}_{i}+\nabla \phi_{i}\right) \in \boldsymbol{L}^{2}(\Omega), \\
\nabla \times \nabla \times\left(\frac{1}{\mu} \nabla \times \boldsymbol{A}_{i}\right)=h_{i} \nabla \times \boldsymbol{f}-\varepsilon \delta^{2} \nabla \times \boldsymbol{A}_{i}-\sigma \delta \nabla \times \boldsymbol{A}_{i} \in \boldsymbol{L}^{2}(\Omega),
\end{gathered}
$$

and

$$
\nabla \times\left(\frac{1}{\mu} \nabla \times \boldsymbol{A}_{i}\right) \times \boldsymbol{n}=h_{i} \boldsymbol{f} \times \boldsymbol{n}-\varepsilon \delta^{2}\left(\boldsymbol{A}_{i}+\nabla \phi_{i}\right) \times \boldsymbol{n}-\sigma \delta\left(\boldsymbol{A}_{i}+\nabla \phi_{i}\right) \times \boldsymbol{n}=\mathbf{0} \text { on } \partial \Omega,
$$

due to the assumption $\boldsymbol{f} \times \boldsymbol{n}=\mathbf{0}$ on the boundary. Moreover, $\boldsymbol{f} \in \widehat{\boldsymbol{H}}_{0}^{1}(\Omega)$ implies that $\boldsymbol{f}$ is well-defined on the interface such that

$$
\left[\nabla \times\left(\frac{1}{\mu} \nabla \times \boldsymbol{A}_{i}\right) \times \boldsymbol{n}_{12}\right]=\mathbf{0} \text { on } \Gamma \text {. }
$$

Putting $\psi=0$ in Lemma 2.1, we get the inequality

$$
\left\|\nabla \times\left(\frac{1}{\mu} \nabla \times A_{i}\right)\right\|_{H^{1}(\Omega)}^{2} \leq C\left(\left\|\nabla \times\left(\frac{1}{\mu} \nabla \times A_{i}\right)\right\|^{2}+\left\|\nabla \times \nabla \times\left(\frac{1}{\mu} \nabla \times A_{i}\right)\right\|^{2}\right),
$$

which means that

$$
\nabla \times\left(\frac{1}{\mu} \nabla \times \boldsymbol{A}_{i}\right) \in \widehat{\boldsymbol{H}}_{0}^{1}(\Omega), \text { i.e. } \nabla \times \nabla \times \boldsymbol{A}_{i} \in \widehat{\boldsymbol{H}}_{0}^{1}(\Omega),
$$

which finish the proof. 
Under the assumptions of Theorem 2.3, the weak form of (2.4)-(2.5) reads as: find $\left(\boldsymbol{A}_{i}, \phi_{i}\right) \in \boldsymbol{V}, 1 \leq i \leq n$, such that

$$
\begin{aligned}
\left(\varepsilon \delta^{2}\left(\boldsymbol{A}_{i}+\nabla \phi_{i}\right), \boldsymbol{Q}+\nabla \psi\right)+\sigma_{c}( & \left.\left(\boldsymbol{A}_{i}+\nabla \phi_{i}\right), \boldsymbol{Q}+\nabla \psi\right)_{\Omega_{c}} \\
& +\left(\frac{1}{\mu} \nabla \times \boldsymbol{A}_{i}, \nabla \times \boldsymbol{Q}\right)+\left(\frac{1}{\mu} \nabla \cdot \boldsymbol{A}_{i}, \nabla \cdot \boldsymbol{Q}\right)=\left(h_{i} \boldsymbol{f}, \boldsymbol{Q}+\nabla \psi\right), \quad \forall(\boldsymbol{Q}, \psi) \in \boldsymbol{V}
\end{aligned}
$$

with

$$
h_{i}=\frac{-\varepsilon_{c} m_{i}^{\prime}-\sigma_{c} m_{i}+\frac{1}{\mu_{c}} \int_{\gamma} \nabla \times \nabla \times \boldsymbol{A}_{i-1} \cdot \boldsymbol{n} d \gamma}{\int_{\gamma} \boldsymbol{f} \cdot \boldsymbol{n} d \gamma} .
$$

Remark 2.2. The term $\left(\frac{1}{\mu} \nabla \cdot \boldsymbol{A}_{i}, \nabla \cdot \boldsymbol{Q}\right)$ is needed in (2.8) to assure that the solution $\boldsymbol{A}_{i}$ of problem (2.8) for given $h_{i}$ is divergence free. This implies (starting from $\nabla \cdot \boldsymbol{A}_{0}=0$ ) that the corresponding term can be deleted in (2.9).

\section{Stability}

In the following lemmas, we give some stability estimates for the solution $\left(\boldsymbol{A}_{i}, \phi_{i}, h_{i}\right)$ in order to prove the wellposedness of the problem.

Lemma 3.1. Let the assumptions of Theorem 2.3 be satisfied. Then there exist positive constants $C$ and $\tau_{0}$ such that for $1 \leq j \leq n$ and for all $0<\tau<\tau_{0}$ holds that

$$
\left\|\boldsymbol{A}_{j}+\nabla \phi_{j}\right\|^{2}+\sum_{i=1}^{j}\left\|\left(\boldsymbol{A}_{i}+\nabla \phi_{i}\right)-\left(\boldsymbol{A}_{i-1}+\nabla \phi_{i-1}\right)\right\|^{2}+\sum_{i=1}^{j}\left\|\tau \nabla \times \boldsymbol{A}_{i}\right\|^{2} \leq C\left(1+\sum_{i=1}^{j} \tau h_{i}^{2}\right) .
$$

Proof. First, we multiply equation (2.7) by $\tau$ and sum up for $i=1, \ldots, k$. If we define the sequence $\boldsymbol{b}_{k}=\sum_{i=1}^{k} \tau \nabla \times \boldsymbol{A}_{i}$ with $\boldsymbol{b}_{0}=\mathbf{0}$, this results in $\left(\nabla \cdot \boldsymbol{A}_{i}=0, \forall i\right)$

$$
\begin{aligned}
\left(\varepsilon \delta\left(\boldsymbol{A}_{k}+\nabla \phi_{k}\right), \boldsymbol{Q}+\nabla \psi\right)+\sigma_{c}\left(\boldsymbol{A}_{k}\right. & \left.+\nabla \phi_{k}, \boldsymbol{Q}+\nabla \psi\right)_{\Omega_{c}}+\left(\frac{1}{\mu} \boldsymbol{b}_{k}, \nabla \times \boldsymbol{Q}\right) \\
& =\left(\sum_{i=1}^{k} \tau h_{i} f, \boldsymbol{Q}+\nabla \psi\right)+\left(\varepsilon\left(\boldsymbol{A}_{0}^{\prime}+\nabla \phi_{0}^{\prime}\right), \boldsymbol{Q}+\nabla \psi\right)+\sigma_{c}\left(\boldsymbol{A}_{0}+\nabla \phi_{0}, \boldsymbol{Q}+\nabla \psi\right)_{\Omega_{c}}
\end{aligned}
$$

for any $(\boldsymbol{Q}, \psi) \in \boldsymbol{V}$. Then we put $(\boldsymbol{Q}, \psi)=\left(\boldsymbol{A}_{k}, \phi_{k}\right)$ and multiply the result by $\tau$ and sum up for $k=1, \ldots, j$. We obtain that

$$
\begin{aligned}
\sum_{k=1}^{j}\left(\varepsilon \delta \left(\boldsymbol{A}_{k}\right.\right. & \left.\left.+\nabla \phi_{k}\right), \boldsymbol{A}_{k}+\nabla \phi_{k}\right) \tau+\sigma_{c} \sum_{k=1}^{j}\left\|\boldsymbol{A}_{k}+\nabla \phi_{k}\right\|_{\boldsymbol{L}^{2}\left(\Omega_{c}\right)}^{2} \tau+\sum_{k=1}^{j}\left(\frac{1}{\mu} \boldsymbol{b}_{k}, \delta \boldsymbol{b}_{k}\right) \tau \\
& =\sum_{k=1}^{j}\left(\sum_{i=1}^{k} \tau h_{i} \boldsymbol{f}, \boldsymbol{A}_{k}+\nabla \phi_{k}\right) \tau+\sum_{k=1}^{j}\left(\varepsilon\left(\boldsymbol{A}_{0}^{\prime}+\nabla \phi_{0}^{\prime}\right), \boldsymbol{A}_{k}+\nabla \phi_{k}\right) \tau+\sum_{k=1}^{j} \sigma_{c}\left(\boldsymbol{A}_{0}+\nabla \phi_{0}, \boldsymbol{A}_{k}+\nabla \phi_{k}\right)_{\Omega_{c}} \tau
\end{aligned}
$$

Next, we multiply this result by $\tau$ again and sum up for $k=1, \cdots, j$. The first and last term on the left-hand side (LHS) of equation (3.3) can be rewritten using Abel's summation rule and estimated as follows:

$$
\begin{aligned}
2 \sum_{k=1}^{j} \tau\left(\varepsilon \delta\left(\boldsymbol{A}_{k}+\nabla \phi_{k}\right),\right. & \left.\boldsymbol{A}_{k}+\nabla \phi_{k}\right) \\
& =\sum_{k=1}^{j}\left\|\sqrt{\varepsilon}\left(\boldsymbol{A}_{k}+\nabla \phi_{k}\right)-\sqrt{\varepsilon}\left(\boldsymbol{A}_{k-1}+\nabla \phi_{k-1}\right)\right\|^{2}+\left\|\sqrt{\varepsilon}\left(\boldsymbol{A}_{j}+\nabla \phi_{j}\right)\right\|^{2}-\left\|\sqrt{\varepsilon}\left(\boldsymbol{A}_{0}+\nabla \phi_{0}\right)\right\|^{2}
\end{aligned}
$$

and

$$
\sum_{k=1}^{j} \tau\left(\frac{1}{\mu} \boldsymbol{b}_{k}, \delta \boldsymbol{b}_{k}\right)=\frac{1}{2} \sum_{k=1}^{j}\left\|\frac{1}{\sqrt{\mu}}\left(\boldsymbol{b}_{k}-\boldsymbol{b}_{k-1}\right)\right\|^{2}+\frac{1}{2}\left\|\frac{1}{\sqrt{\mu}} \boldsymbol{b}_{j}\right\|^{2}-\frac{1}{2}\left\|\frac{1}{\sqrt{\mu}} \boldsymbol{b}_{0}\right\|^{2} .
$$


The second term on the LHS of equation (3.3) is positive and can be omitted. For the first term on the right-hand side (RHS), we apply Cauchy's and Young's inequalities together with the assumptions on the source function to get that

$$
\left|\sum_{k=1}^{j}\left(\sum_{i=1}^{k} \tau h_{i} \boldsymbol{f}, \boldsymbol{A}_{k}+\nabla \phi_{k}\right) \tau\right| \leq C \sum_{i=1}^{j} h_{i}^{2} \tau+C \sum_{k=1}^{j}\left\|\boldsymbol{A}_{k}+\nabla \phi_{k}\right\|^{2} \tau
$$

The second and third term on the RHS is easily bounded using Cauchy's and Young's inequalities as follows

$$
\begin{aligned}
\left|\sum_{k=1}^{j}\left(\varepsilon\left(\boldsymbol{A}_{0}^{\prime}+\nabla \phi_{0}^{\prime}\right), \boldsymbol{A}_{k}+\nabla \phi_{k}\right) \tau\right| & \leq C+C \sum_{k=1}^{j}\left\|\boldsymbol{A}_{k}+\nabla \phi_{k}\right\|^{2} \tau, \\
\left|\sum_{k=1}^{j} \sigma_{c}\left(\boldsymbol{A}_{0}+\nabla \phi_{0}, \boldsymbol{A}_{k}+\nabla \phi_{k}\right)_{\Omega_{c}} \tau\right| & \leq C+C \sum_{k=1}^{j}\left\|\boldsymbol{A}_{k}+\nabla \phi_{k}\right\|^{2} \tau .
\end{aligned}
$$

Finally, we arrive at the following inequality (change index $k \leftrightarrow i$ ):

$$
\begin{aligned}
\left\|\boldsymbol{A}_{j}+\nabla \phi_{j}\right\|^{2}+\sum_{i=1}^{j}\left\|\left(\boldsymbol{A}_{i}+\nabla \phi_{i}\right)-\left(\boldsymbol{A}_{i-1}+\nabla \phi_{i-1}\right)\right\|^{2} & \left\|\sum_{i=1}^{j} \tau \nabla \times \boldsymbol{A}_{i}\right\|^{2} \\
& +\sum_{i=1}^{j}\left\|\tau \nabla \times \boldsymbol{A}_{i}\right\|^{2} \leq C+C \sum_{i=1}^{j} h_{i}^{2} \tau+C \sum_{i=1}^{j}\left\|\boldsymbol{A}_{i}+\nabla \phi_{i}\right\|^{2} \tau .
\end{aligned}
$$

An application of Grönwall's inequality concludes the proof.

Lemma 3.2. Let the assumptions of Lemma 3.1 be satisfied. Then there exist positive constants $C$ and $\tau_{0}$ such that for $1 \leq j \leq n$ and for all $0<\tau<\tau_{0}$ holds that

$$
\left\|\delta\left(\boldsymbol{A}_{j}+\nabla \phi_{j}\right)\right\|^{2}+\sum_{i=1}^{j}\left\|\delta\left(\boldsymbol{A}_{i}+\nabla \phi_{i}\right)-\delta\left(\boldsymbol{A}_{i-1}+\nabla \phi_{i-1}\right)\right\|^{2}+\left\|\nabla \times \boldsymbol{A}_{j}\right\|^{2}+\sum_{i=1}^{j}\left\|\nabla \times\left(\boldsymbol{A}_{i}-\boldsymbol{A}_{i-1}\right)\right\|^{2} \leq C\left(1+\sum_{i=1}^{j} \tau h_{i}^{2}\right) .
$$

Proof. Setting $(\boldsymbol{Q}, \psi)=\tau\left(\delta \boldsymbol{A}_{i}, \delta \phi_{i}\right)$ in equation (2.7) and summing up for $i=1, \ldots, j$ lead to

$$
\sum_{i=1}^{j} \tau\left(\varepsilon \delta^{2}\left(\boldsymbol{A}_{i}+\nabla \phi_{i}\right), \delta\left(\boldsymbol{A}_{i}+\nabla \phi_{i}\right)\right)+\sigma_{c} \sum_{i=1}^{j} \tau\left\|\delta\left(\boldsymbol{A}_{i}+\nabla \phi_{i}\right)\right\|_{L^{2}\left(\Omega_{c}\right)}^{2}+\sum_{i=1}^{j} \tau\left(\frac{1}{\mu} \nabla \times \boldsymbol{A}_{i}, \nabla \times \delta \boldsymbol{A}_{i}\right)=\sum_{i=1}^{j} \tau\left(h_{i} f, \delta\left(\boldsymbol{A}_{i}+\nabla \phi_{i}\right)\right) .
$$

We also apply Abel's summation rule to the first and third term to have

$$
\begin{aligned}
\sum_{i=1}^{j} \tau\left(\varepsilon \delta^{2}\left(\boldsymbol{A}_{i}+\nabla \phi_{i}\right), \delta\left(\boldsymbol{A}_{i}+\nabla \phi_{i}\right)\right)=\frac{1}{2} \sum_{i=1}^{j}\left\|\sqrt{\varepsilon} \delta\left(\boldsymbol{A}_{i}+\nabla \phi_{i}\right)-\sqrt{\varepsilon} \delta\left(\boldsymbol{A}_{i-1}+\nabla \phi_{i-1}\right)\right\|^{2} & \\
& +\frac{1}{2}\left\|\sqrt{\varepsilon} \delta\left(\boldsymbol{A}_{j}+\nabla \phi_{j}\right)\right\|^{2}-\frac{1}{2}\left\|\sqrt{\varepsilon}\left(\boldsymbol{A}_{0}^{\prime}+\nabla \phi_{0}^{\prime}\right)\right\|^{2}
\end{aligned}
$$

and

$$
\sum_{i=1}^{j} \tau\left(\frac{1}{\mu} \nabla \times \boldsymbol{A}_{i}, \nabla \times \delta \boldsymbol{A}_{i}\right)=\frac{1}{2} \sum_{i=1}^{j}\left\|\frac{1}{\sqrt{\mu}} \nabla \times\left(\boldsymbol{A}_{i}-\boldsymbol{A}_{i-1}\right)\right\|^{2}+\frac{1}{2}\left\|\frac{1}{\sqrt{\mu}} \nabla \times \boldsymbol{A}_{j}\right\|^{2}-\frac{1}{2}\left\|\frac{1}{\sqrt{\mu}} \nabla \times \boldsymbol{A}_{0}\right\|^{2} .
$$

For the RHS of equation (3.5), we use Cauchy's and Young's inequalities to obtain

$$
\left|\sum_{i=1}^{j} \tau\left(h_{i} f, \delta\left(\boldsymbol{A}_{i}+\nabla \phi_{i}\right)\right)\right| \leq C \sum_{i=1}^{j} \tau h_{i}^{2}+C \sum_{i=1}^{j} \tau\left\|\delta\left(\boldsymbol{A}_{i}+\nabla \phi_{i}\right)\right\|^{2} .
$$

Eventually, we arrive at the following inequality:

$$
\begin{aligned}
\left\|\delta\left(\boldsymbol{A}_{j}+\nabla \phi_{j}\right)\right\|^{2}+\sum_{i=1}^{j} \| \delta\left(\boldsymbol{A}_{i}+\nabla \phi_{i}\right)-\delta\left(\boldsymbol{A}_{i-1}\right. & \left.+\nabla \phi_{i-1}\right)\left\|^{2}+\right\| \nabla \times \boldsymbol{A}_{j} \|^{2} \\
& +\sum_{i=1}^{j}\left\|\nabla \times\left(\boldsymbol{A}_{i}-\boldsymbol{A}_{i-1}\right)\right\|^{2} \leq C+C \sum_{i=1}^{j} \tau h_{i}^{2}+C \sum_{i=1}^{j} \tau\left\|\delta\left(\boldsymbol{A}_{i}+\nabla \phi_{i}\right)\right\|^{2} .
\end{aligned}
$$

62 Applying Grönwall's inequality, we conclude the inequality (3.4). 
Lemma 3.3. Let the assumptions of Lemma 3.2 be satisfied. Moreover, assume that $\nabla \times \nabla \times f \in \boldsymbol{L}^{2}(\Omega)$ and $\nabla \times \nabla \times \boldsymbol{A}_{0}^{\prime} \in$ $\boldsymbol{L}^{2}(\Omega)$. Then there exist positive constants $C$ and $\tau_{0}$ such that for $1 \leq j \leq n$ and for all $0<\tau<\tau_{0}$ holds that

$$
\left\|\nabla \times \nabla \times \boldsymbol{A}_{j}\right\|^{2}+\sum_{i=1}^{j}\left\|\nabla \times \nabla \times\left(\boldsymbol{A}_{i}-\boldsymbol{A}_{i-1}\right)\right\|^{2}+\sum_{i=1}^{j}\left\|\tau \nabla \times \nabla \times \nabla \times \boldsymbol{A}_{i}\right\|^{2} \leq C+C \sum_{i=1}^{j} h_{i}^{2} \tau .
$$

Proof. First, applying the curl operator to both sides of the first equation of (2.4) yields

$$
\nabla \times\left(\varepsilon \delta^{2} \boldsymbol{A}_{i}\right)+\nabla \times\left(\sigma \delta \boldsymbol{A}_{i}\right)+\nabla \times \nabla \times\left(\frac{1}{\mu} \nabla \times \boldsymbol{A}_{i}\right)=h_{i} \nabla \times \boldsymbol{f} .
$$

We multiply this by $\tau$ again and sum up for $i=1, \cdots, k$ to obtain

$$
\nabla \times\left(\varepsilon \delta \boldsymbol{A}_{k}\right)+\nabla \times\left(\sigma \boldsymbol{A}_{k}\right)+\sum_{i=1}^{k} \tau \nabla \times \nabla \times\left(\frac{1}{\mu} \nabla \times \boldsymbol{A}_{i}\right)=\sum_{i=1}^{k} \tau h_{i} \nabla \times \boldsymbol{f}+\nabla \times\left(\varepsilon \boldsymbol{A}_{0}^{\prime}\right)+\nabla \times\left(\sigma \boldsymbol{A}_{0}\right) .
$$

Then taking the scalar product with $\nabla \times \nabla \times\left(\frac{1}{\mu} \nabla \times \boldsymbol{A}_{k}\right)$ and integrating over $\Omega$, we have that

$$
\begin{aligned}
\left(\nabla \times\left(\varepsilon \delta \boldsymbol{A}_{k}\right), \nabla \times \nabla \times\left(\frac{1}{\mu} \nabla \times \boldsymbol{A}_{k}\right)\right) & +\left(\nabla \times\left(\sigma \boldsymbol{A}_{k}\right), \nabla \times \nabla \times\left(\frac{1}{\mu} \nabla \times \boldsymbol{A}_{k}\right)\right) \\
+\left(\sum_{i=1}^{k} \tau \nabla \times \nabla \times\left(\frac{1}{\mu} \nabla \times \boldsymbol{A}_{i}\right), \nabla \times\right. & \left.\nabla \times\left(\frac{1}{\mu} \nabla \times \boldsymbol{A}_{k}\right)\right)=\left(\sum_{i=1}^{k} \tau h_{i} \nabla \times \boldsymbol{f}, \nabla \times \nabla \times\left(\frac{1}{\mu} \nabla \times \boldsymbol{A}_{k}\right)\right) \\
+ & \left(\nabla \times\left(\varepsilon \boldsymbol{A}_{0}^{\prime}\right), \nabla \times \nabla \times\left(\frac{1}{\mu} \nabla \times \boldsymbol{A}_{k}\right)\right)+\left(\nabla \times\left(\sigma \boldsymbol{A}_{0}\right), \nabla \times \nabla \times\left(\frac{1}{\mu} \nabla \times \boldsymbol{A}_{k}\right)\right) .
\end{aligned}
$$

Next, multiplying (3.9) by $\tau$ again and summing up for $k=1, \cdots, j$ lead to

$$
\begin{aligned}
\sum_{k=1}^{j} \tau\left(\nabla \times\left(\varepsilon \delta \boldsymbol{A}_{k}\right), \nabla \times\right. & \left.\nabla \times\left(\frac{1}{\mu} \nabla \times \boldsymbol{A}_{k}\right)\right)+\sum_{k=1}^{j} \tau\left(\nabla \times\left(\sigma \boldsymbol{A}_{k}\right), \nabla \times \nabla \times\left(\frac{1}{\mu} \nabla \times \boldsymbol{A}_{k}\right)\right) \\
+\sum_{k=1}^{j} \tau\left(\sum_{i=1}^{k} \tau \nabla \times \nabla \times(\right. & \left.\left(\frac{1}{\mu} \nabla \times \boldsymbol{A}_{i}\right), \nabla \times \nabla \times\left(\frac{1}{\mu} \nabla \times \boldsymbol{A}_{k}\right)\right)=\sum_{k=1}^{j} \tau\left(\sum_{i=1}^{k} \tau h_{i} \nabla \times \boldsymbol{f}, \nabla \times \nabla \times\left(\frac{1}{\mu} \nabla \times \boldsymbol{A}_{k}\right)\right) \\
& +\sum_{k=1}^{j} \tau\left(\nabla \times\left(\varepsilon \boldsymbol{A}_{0}^{\prime}\right), \nabla \times \nabla \times\left(\frac{1}{\mu} \nabla \times \boldsymbol{A}_{k}\right)\right)+\sum_{k=1}^{j} \tau\left(\nabla \times\left(\sigma \boldsymbol{A}_{0}\right), \nabla \times \nabla \times\left(\frac{1}{\mu} \nabla \times \boldsymbol{A}_{k}\right)\right) .
\end{aligned}
$$

We apply Abel's summation rule to the third term on the LHS of (3.10) to get

$$
\begin{aligned}
& \sum_{k=1}^{j} \tau\left(\sum_{i=1}^{k} \tau \nabla \times \nabla \times\left(\frac{1}{\mu} \nabla \times \boldsymbol{A}_{i}\right), \nabla \times \nabla \times\left(\frac{1}{\mu} \nabla \times \boldsymbol{A}_{k}\right)\right) \\
& =\sum_{k=1}^{j} \frac{\tau}{\mu_{c}^{2}}\left(\sum_{i=1}^{k} \tau \nabla \times \nabla \times \nabla \times \boldsymbol{A}_{i}, \nabla \times \nabla \times \nabla \times \boldsymbol{A}_{k}\right){ }_{\Omega_{c}}+\sum_{k=1}^{j} \frac{\tau}{\mu_{e}^{2}}\left(\sum_{i=1}^{k} \tau \nabla \times \nabla \times \nabla \times \boldsymbol{A}_{i}, \nabla \times \nabla \times \nabla \times \boldsymbol{A}_{k}\right)_{\Omega_{e}} \\
& =\frac{1}{2 \mu_{c}^{2}} \sum_{k=1}^{j}\left\|\tau \nabla \times \nabla \times \nabla \times \boldsymbol{A}_{k}\right\|_{\mathbf{L}^{2}\left(\Omega_{c}\right)}^{2}+\frac{1}{2 \mu_{c}^{2}}\left\|\sum_{k=1}^{j} \tau \nabla \times \nabla \times \nabla \times \boldsymbol{A}_{k}\right\|_{\mathbf{L}^{2}\left(\Omega_{c}\right)}^{2} \\
& +\frac{1}{2 \mu_{e}^{2}} \sum_{k=1}^{j}\left\|\tau \nabla \times \nabla \times \nabla \times \boldsymbol{A}_{k}\right\|_{\mathbf{L}^{2}\left(\Omega_{e}\right)}^{2}+\frac{1}{2 \mu_{e}^{2}}\left\|\sum_{k=1}^{j} \tau \nabla \times \nabla \times \nabla \times \boldsymbol{A}_{k}\right\|_{\boldsymbol{L}^{2}\left(\Omega_{e}\right)}^{2} \\
& \geq \frac{1}{2 \mu_{\max }^{2}}\left\{\sum_{k=1}^{j}\left\|\tau \nabla \times \nabla \times \nabla \times \boldsymbol{A}_{k}\right\|^{2}+\left\|\sum_{k=1}^{j} \tau \nabla \times \nabla \times \nabla \times \boldsymbol{A}_{k}\right\|^{2}\right\} .
\end{aligned}
$$


Since $\nabla \times\left(\frac{1}{\mu} \nabla \times \boldsymbol{A}_{k}\right) \times \boldsymbol{n}=\mathbf{0}$ on $\partial \Omega$ and $\left[\nabla \times\left(\frac{1}{\mu} \nabla \times \boldsymbol{A}_{k}\right) \times \boldsymbol{n}\right]=\mathbf{0}$ on $\Gamma$, we have that

$$
\begin{aligned}
& \sum_{k=1}^{j} \tau\left(\nabla \times\left(\varepsilon \delta \boldsymbol{A}_{k}\right), \nabla \times \nabla \times\left(\frac{1}{\mu} \nabla \times \boldsymbol{A}_{k}\right)\right)_{\Omega_{e} \cup \Omega_{c}} \\
& =\sum_{k=1}^{j} \tau\left(\nabla \times \nabla \times\left(\varepsilon \delta \boldsymbol{A}_{k}\right), \nabla \times\left(\frac{1}{\mu} \nabla \times \boldsymbol{A}_{k}\right)\right)_{\Omega_{e} \cup \Omega_{c}} \\
& =\sum_{k=1}^{j} \tau \frac{\varepsilon_{c}}{\mu_{c}}\left(\nabla \times \nabla \times\left(\delta \boldsymbol{A}_{k}\right), \nabla \times \nabla \times \boldsymbol{A}_{k}\right)_{\Omega_{c}}+\sum_{k=1}^{j} \tau \frac{\varepsilon_{e}}{\mu_{e}}\left(\nabla \times \nabla \times\left(\delta \boldsymbol{A}_{k}\right), \nabla \times \nabla \times \boldsymbol{A}_{k}\right)_{\Omega_{e}} \\
& =\frac{\varepsilon_{c}}{2 \mu_{c}}\left(\left\|\nabla \times \nabla \times \boldsymbol{A}_{j}\right\|_{L^{2}\left(\Omega_{c}\right)}^{2}-\left\|\nabla \times \nabla \times \boldsymbol{A}_{0}\right\|_{L^{2}\left(\Omega_{c}\right)}^{2}+\sum_{k=1}^{j}\left\|\nabla \times \nabla \times\left(\boldsymbol{A}_{k}-\boldsymbol{A}_{k-1}\right)\right\|_{\boldsymbol{L}^{2}\left(\Omega_{c}\right)}^{2}\right) \\
& +\frac{\varepsilon_{e}}{2 \mu_{e}}\left(\left\|\nabla \times \nabla \times \boldsymbol{A}_{j}\right\|_{\boldsymbol{L}^{2}\left(\Omega_{e}\right)}^{2}-\left\|\nabla \times \nabla \times \boldsymbol{A}_{0}\right\|_{\boldsymbol{L}^{2}\left(\Omega_{e}\right)}^{2}+\sum_{k=1}^{j}\left\|\nabla \times \nabla \times\left(\boldsymbol{A}_{k}-\boldsymbol{A}_{k-1}\right)\right\|_{\boldsymbol{L}^{2}\left(\Omega_{e}\right)}^{2}\right) \\
& \geq \frac{\varepsilon_{\min }}{2 \mu_{\max }}\left\|\nabla \times \nabla \times \boldsymbol{A}_{j}\right\|^{2}-\frac{\varepsilon_{\max }}{2 \mu_{\min }}\left\|\nabla \times \nabla \times \boldsymbol{A}_{0}\right\|^{2}+\frac{\varepsilon_{\min }}{2 \mu_{\max }} \sum_{k=1}^{j}\left\|\nabla \times \nabla \times\left(\boldsymbol{A}_{k}-\boldsymbol{A}_{k-1}\right)\right\|^{2} .
\end{aligned}
$$

Analogously, we obtain that

$$
\sum_{k=1}^{j} \tau\left(\nabla \times\left(\sigma \boldsymbol{A}_{k}\right), \nabla \times \nabla \times\left(\frac{1}{\mu} \nabla \times \boldsymbol{A}_{k}\right)\right)=\frac{\sigma_{c}}{\mu_{c}} \sum_{k=1}^{j}\left\|\nabla \times \nabla \times \boldsymbol{A}_{k}\right\|_{L^{2}\left(\Omega_{c}\right)}^{2} \tau \geq 0 .
$$

For the terms on the RHS of (3.10), we use Green's theorem, Cauchy's and Young's inequalities together with the assumptions $\nabla \times \nabla \times f \in \boldsymbol{L}^{2}(\Omega)$ and $\nabla \times \nabla \times \boldsymbol{A}_{0}^{\prime} \in \boldsymbol{L}^{2}(\Omega)$ to obtain that

$$
\begin{aligned}
& \left|\sum_{k=1}^{j} \tau\left(\sum_{i=1}^{k} \tau h_{i} \nabla \times f, \nabla \times \nabla \times\left(\frac{1}{\mu} \nabla \times \boldsymbol{A}_{k}\right)\right)\right| \\
& =\left|\sum_{k=1}^{j} \tau\left(\sum_{i=1}^{k} \tau h_{i} \nabla \times \nabla \times f, \nabla \times\left(\frac{1}{\mu} \nabla \times \boldsymbol{A}_{k}\right)\right)\right| \\
& \leq \frac{1}{2} \sum_{k=1}^{j}\left\|\sum_{i=1}^{k} \tau h_{i} \nabla \times \nabla \times f||^{2} \tau+\frac{1}{2} \sum_{k=1}^{j}\right\| \nabla \times\left(\frac{1}{\mu} \nabla \times \boldsymbol{A}_{k}\right) \|^{2} \tau \\
& \leq \frac{1}{2} \sum_{k=1}^{j}\left(\sum_{i=1}^{k} \tau\left|h_{i}\right| \| \nabla \times \nabla \times f\right)^{2} \tau+\frac{1}{2 \mu_{e}} \sum_{k=1}^{j}\left\|\nabla \times \nabla \times \boldsymbol{A}_{k}\right\|_{\boldsymbol{L}^{2}\left(\Omega_{e}\right)}^{2} \tau+\frac{1}{2 \mu_{c}} \sum_{k=1}^{j}\left\|\nabla \times \nabla \times \boldsymbol{A}_{k}\right\|_{\mathbf{L}^{2}\left(\Omega_{c}\right)}^{2} \tau \\
& \leq C \sum_{k=1}^{j} h_{k}^{2} \tau+C \sum_{k=1}^{j}\left\|\nabla \times \nabla \times \boldsymbol{A}_{k}\right\|^{2} \tau, \quad \leq C+C \sum_{k=1}^{j}\left\|\nabla \times \nabla \times \boldsymbol{A}_{k}\right\|^{2} \tau . \\
& \left|\sum_{k=1}^{j} \tau\left(\nabla \times\left(\varepsilon \boldsymbol{A}_{0}^{\prime}\right), \nabla \times \nabla \times\left(\frac{1}{\mu} \nabla \times \boldsymbol{A}_{k}\right)\right)\right|=\left|\sum_{k=1}^{j} \tau\left(\nabla \times \nabla \times\left(\varepsilon \boldsymbol{A}_{0}^{\prime}\right), \nabla \times\left(\frac{1}{\mu} \nabla \times \boldsymbol{A}_{k}\right)\right)\right| \\
& \left|\sum_{k=1}^{j} \tau\left(\nabla \times\left(\sigma \boldsymbol{A}_{0}\right), \nabla \times \nabla \times\left(\frac{1}{\mu} \nabla \times \boldsymbol{A}_{k}\right)\right)\right|=\left|\sum_{k=1}^{j} \tau\left(\nabla \times \nabla \times\left(\sigma \boldsymbol{A}_{0}\right), \nabla \times\left(\frac{1}{\mu} \nabla \times \boldsymbol{A}_{k}\right)\right)\right| \\
& \leq
\end{aligned}
$$


Finally, after collecting all estimates above, we receive that

$$
\begin{aligned}
\left\|\nabla \times \nabla \times \boldsymbol{A}_{j}\right\|^{2}+\sum_{k=1}^{j}\left\|\nabla \times \nabla \times\left(\boldsymbol{A}_{k}-\boldsymbol{A}_{k-1}\right)\right\|^{2}+\sum_{k=1}^{j}\left\|\tau \nabla \times \nabla \times \nabla \times \boldsymbol{A}_{k}\right\|^{2} \\
+\left\|\sum_{k=1}^{j} \tau \nabla \times \nabla \times \nabla \times \boldsymbol{A}_{k}\right\|^{2} \leq C+C \sum_{k=1}^{j} h_{k}^{2} \tau+C \sum_{k=1}^{j}\left\|\nabla \times \nabla \times \boldsymbol{A}_{k}\right\|^{2} \tau .
\end{aligned}
$$

An application of Grönwall's inequality concludes the proof.

Lemma 3.4. Let the assumptions of Lemma 3.3 be satisfied. Then there exist positive constants $C$ and $\tau_{0}$ such that for $1 \leq j \leq n$ and for all $0<\tau<\tau_{0}$ holds that

$$
\begin{aligned}
\left\|\nabla \times \nabla \times \delta \boldsymbol{A}_{j}\right\|^{2}+\sum_{i=1}^{j} \| \nabla \times \nabla \times & \left(\delta \boldsymbol{A}_{i}-\delta \boldsymbol{A}_{i-1}\right) \|^{2} \\
& +\left\|\nabla \times \nabla \times \nabla \times \boldsymbol{A}_{j}\right\|^{2}+\sum_{i=1}^{j}\left\|\nabla \times \nabla \times \nabla \times\left(\boldsymbol{A}_{i}-\boldsymbol{A}_{i-1}\right)\right\|^{2} \leq C+C \sum_{i=1}^{j} h_{i}^{2} \tau .
\end{aligned}
$$

Proof. First, we take the scalar product of equation (3.7) with $\nabla \times \nabla \times\left(\frac{1}{\mu} \nabla \times \delta \boldsymbol{A}_{i}\right)$ and integrate over $\Omega$. Then, we multiply the result by $\tau$ and sum it up for $k=1, \cdots, j$, i.e.

$$
\begin{aligned}
\sum_{i=1}^{j} \tau\left(\nabla \times\left(\varepsilon \delta^{2} \boldsymbol{A}_{i}\right)\right. & \left., \nabla \times \nabla \times\left(\frac{1}{\mu} \nabla \times \delta \boldsymbol{A}_{i}\right)\right)+\sum_{i=1}^{j} \tau\left(\nabla \times\left(\sigma \delta \boldsymbol{A}_{i}\right), \nabla \times \nabla \times\left(\frac{1}{\mu} \nabla \times \delta \boldsymbol{A}_{i}\right)\right) \\
& +\sum_{i=1}^{j} \tau\left(\nabla \times \nabla \times\left(\frac{1}{\mu} \nabla \times \boldsymbol{A}_{i}\right), \nabla \times \nabla \times\left(\frac{1}{\mu} \nabla \times \delta \boldsymbol{A}_{i}\right)\right)=\sum_{i=1}^{j} \tau\left(h_{i} \nabla \times \boldsymbol{f}, \nabla \times \nabla \times\left(\frac{1}{\mu} \nabla \times \delta \boldsymbol{A}_{i}\right)\right)
\end{aligned}
$$

Note that also $\nabla \times\left(\frac{1}{\mu} \nabla \times \delta \boldsymbol{A}_{i}\right) \times \boldsymbol{n}=\mathbf{0}$ on $\partial \Omega$ and $\left[\nabla \times\left(\frac{1}{\mu} \nabla \times \delta \boldsymbol{A}_{i}\right) \times \boldsymbol{n}_{12}\right]=\mathbf{0}$ on $\Gamma$. For this, the following compatibility condition needs to be defined:

$$
\varepsilon\left(\delta^{2} A_{0}+\nabla \delta^{2} \phi_{0}\right):=h(0) \boldsymbol{f}-\nabla \times\left(\frac{1}{\mu} \nabla \times \boldsymbol{A}_{0}\right)-\sigma\left(\boldsymbol{A}_{0}^{\prime}+\nabla \phi_{0}^{\prime}\right) \in \widehat{\boldsymbol{H}}_{0}^{1}(\Omega)
$$

We apply Abel's summation rule to respectively the first and third term on the LHS of (3.13) to get that

$$
\begin{aligned}
& \sum_{i=1}^{j} \tau\left(\nabla \times\left(\varepsilon \delta^{2} \boldsymbol{A}_{i}\right), \nabla \times \nabla \times\left(\frac{1}{\mu} \nabla \times \delta \boldsymbol{A}_{i}\right)\right)_{\Omega_{e} \cup \Omega_{c}} \\
& =\sum_{i=1}^{j} \tau\left(\nabla \times \nabla \times\left(\varepsilon \delta^{2} \boldsymbol{A}_{i}\right), \nabla \times\left(\frac{1}{\mu} \nabla \times \delta \boldsymbol{A}_{i}\right)\right)_{\Omega_{e} \cup \Omega_{c}} \\
& \geq \frac{\varepsilon_{\min }}{2 \mu_{\max }} \sum_{i=1}^{j}\left\|\nabla \times \nabla \times\left(\delta \boldsymbol{A}_{i}-\delta \boldsymbol{A}_{i-1}\right)\right\|^{2}+\frac{\varepsilon_{\min }}{2 \mu_{\max }}\left\|\nabla \times \nabla \times \delta \boldsymbol{A}_{j}\right\|^{2}-\frac{\varepsilon_{\max }}{2 \mu_{\min }}\left\|\nabla \times \nabla \times \boldsymbol{A}_{0}^{\prime}\right\|^{2}
\end{aligned}
$$

and

$$
\begin{aligned}
& \sum_{i=1}^{j} \tau\left(\nabla \times \nabla \times\left(\frac{1}{\mu} \nabla \times \boldsymbol{A}_{i}\right), \nabla \times \nabla \times\left(\frac{1}{\mu} \nabla \times \delta \boldsymbol{A}_{i}\right)\right) \\
& \geq \frac{1}{2 \mu_{\max }} \sum_{i=1}^{j}\left\|\nabla \times \nabla \times \nabla \times\left(\boldsymbol{A}_{i}-\boldsymbol{A}_{i-1}\right)\right\|^{2}+\frac{1}{2 \mu_{\max }}\left\|\nabla \times \nabla \times \nabla \times \boldsymbol{A}_{j}\right\|^{2}-\frac{1}{2 \mu_{\min }}\left\|\nabla \times \nabla \times \nabla \times \boldsymbol{A}_{0}\right\|^{2} .
\end{aligned}
$$

The second term on the LHS of (3.13) simplifies to

$$
\sum_{i=1}^{j} \tau\left(\nabla \times\left(\sigma \delta \boldsymbol{A}_{i}\right), \nabla \times \nabla \times\left(\frac{1}{\mu} \nabla \times \delta \boldsymbol{A}_{i}\right)\right)=\frac{\sigma_{c}}{\mu_{c}} \sum_{i=1}^{j}\left\|\nabla \times \nabla \times \delta \boldsymbol{A}_{i}\right\|_{L^{2}\left(\Omega_{c}\right)}^{2} \tau \geq 0 .
$$


For the term on the RHS, we use the Cauchy's and Young's inequalities to obtain

$$
\begin{aligned}
\left|\sum_{i=1}^{j} \tau\left(h_{i} \nabla \times f, \nabla \times \nabla \times\left(\frac{1}{\mu} \nabla \times \delta \boldsymbol{A}_{i}\right)\right)\right| & =\left|\sum_{i=1}^{j} \tau\left(h_{i} \nabla \times \nabla \times f, \nabla \times\left(\frac{1}{\mu} \nabla \times \delta \boldsymbol{A}_{i}\right)\right)\right| \\
& \leq C \sum_{i=1}^{j} h_{i}^{2} \tau+C \sum_{i=1}^{j}\left\|\nabla \times \nabla \times \delta \boldsymbol{A}_{i}\right\|^{2} \tau,
\end{aligned}
$$

64 Combining all the previous estimates and applying Grönwall's inequality concludes the proof.

Theorem 3.5. Let the assumptions of Lemma 3.4 be satisfied. Then there exist positive constants $C$ and $\tau_{0}$ such that for all $0<\tau<\tau_{0}$ holds that

$$
\begin{aligned}
& \sum_{i=1}^{n} h_{i}^{2} \tau \leq C \\
& \max _{1 \leq j \leq n}\left\|\boldsymbol{A}_{j}+\nabla \phi_{j}\right\|^{2}+\sum_{i=1}^{n}\left\|\left(\boldsymbol{A}_{i}+\nabla \phi_{i}\right)-\left(\boldsymbol{A}_{i-1}+\nabla \phi_{i-1}\right)\right\|^{2} \leq C \\
& \max _{1 \leq j \leq n}\left\|\delta\left(\boldsymbol{A}_{j}+\nabla \phi_{j}\right)\right\|^{2}+\sum_{i=1}^{n}\left\|\delta\left(\boldsymbol{A}_{i}+\nabla \phi_{i}\right)-\delta\left(\boldsymbol{A}_{i-1}+\nabla \phi_{i-1}\right)\right\|^{2}+\max _{1 \leq j \leq n}\left\|\nabla \times \boldsymbol{A}_{j}\right\|^{2}+\sum_{i=1}^{n}\left\|\nabla \times\left(\boldsymbol{A}_{i}-\boldsymbol{A}_{i-1}\right)\right\|^{2} \leq C, \\
& \max _{1 \leq j \leq n}\left\|\nabla \times \nabla \times \boldsymbol{A}_{j}\right\|^{2}+\sum_{i=1}^{n}\left\|\nabla \times \nabla \times\left(\boldsymbol{A}_{i}-\boldsymbol{A}_{i-1}\right)\right\|^{2} \leq C
\end{aligned}
$$

and

$$
\begin{aligned}
\max _{1 \leq j \leq n}\left\|\nabla \times \nabla \times \delta \boldsymbol{A}_{j}\right\|^{2}+\sum_{i=1}^{n}\left\|\nabla \times \nabla \times\left(\delta \boldsymbol{A}_{i}-\delta \boldsymbol{A}_{i-1}\right)\right\|^{2} \\
+\max _{1 \leq j \leq n}\left\|\nabla \times \nabla \times \nabla \times \boldsymbol{A}_{j}\right\|^{2}+\sum_{i=1}^{n}\left\|\nabla \times \nabla \times \nabla \times\left(\boldsymbol{A}_{i}-\boldsymbol{A}_{i-1}\right)\right\|^{2} \leq C .
\end{aligned}
$$

Proof. Starting from equation (2.9), we follow the same steps as in Theorem 2.3 to obtain that

$$
\begin{aligned}
\sum_{i=1}^{j} h_{i}^{2} \tau & =\sum_{i=1}^{n}\left(\frac{-\varepsilon_{c} m_{i}^{\prime}-\sigma_{c} m_{i}+\frac{1}{\mu_{c}} \int_{\gamma} \nabla \times \nabla \times \boldsymbol{A}_{i-1} \cdot \boldsymbol{n} d \gamma}{\int_{\gamma} \boldsymbol{f} \cdot \boldsymbol{n} d \gamma}\right)^{2} \tau \\
& \leq C \sum_{i=1}^{j}\left(1+\left\|\nabla \times \nabla \times \boldsymbol{A}_{i-1}\right\|_{\boldsymbol{L}^{2}(\gamma)}^{2}\right) \tau \\
& \leq C+C \sum_{i=1}^{j}\left\|\nabla \times \nabla \times \boldsymbol{A}_{i-1}\right\|_{\boldsymbol{H}^{1}(G)}^{2} \boldsymbol{\tau} \\
& \leq C+C \sum_{i=1}^{j-1}\left(\left\|\nabla \times \nabla \times \boldsymbol{A}_{i}\right\|^{2}+\left\|\nabla \times \nabla \times \nabla \times \boldsymbol{A}_{i}\right\|^{2}\right) \tau .
\end{aligned}
$$

From Lemma 3.3 and Lemma 3.4 follows that

$$
\sum_{i=1}^{j} h_{i}^{2} \tau \leq C+\sum_{i=1}^{j-1}\left(\sum_{k=1}^{i} h_{k}^{2} \tau\right) \tau .
$$

Again an application of Grönwall's lemma gives that

$$
\sum_{i=1}^{j} h_{i}^{2} \tau \leq C
$$

65 The other results of this lemma follow now easily from Lemma 3.1-3.4. 
Corollary 3.1. Let the assumptions of Theorem 3.5 be fulfilled. Then there exist positive constants $C$ and $\tau_{0}$ such that for all $0<\tau<\tau_{0}$ holds that

$$
\max _{1 \leq j \leq n}\left\{\left\|\boldsymbol{A}_{j}\right\|_{\boldsymbol{H}^{1}(\Omega)}^{2}+\left\|\phi_{j}\right\|_{H^{1}(\Omega)}^{2}+\left\|\nabla \times \nabla \times \boldsymbol{A}_{j}\right\|_{\boldsymbol{H}^{1}(\Omega)}^{2}\right\}+\sum_{i=1}^{n}\left\|\boldsymbol{A}_{i}-\boldsymbol{A}_{i-1}\right\|_{\boldsymbol{H}^{1}(\Omega)}^{2}+\sum_{i=1}^{n}\left\|\phi_{i}-\phi_{i-1}\right\|_{H^{1}(\Omega)}^{2} \leq C .
$$

66 Proof. The result is a consequence of Theorem 3.5 and Lemma 2.1.

Corollary 3.2. Let the assumptions of Theorem 3.5 be fulfilled. Then there exist positive constants $C$ and $\tau_{0}$ such that for all $0<\tau<\tau_{0}$ holds that

$$
\max _{1 \leq j \leq n}\left\|\nabla \times \delta \boldsymbol{A}_{j}\right\|_{L^{2}\left(\Omega_{c}\right)}^{2}+\sum_{i=1}^{n}\left\|\nabla \times\left(\delta \boldsymbol{A}_{i}-\delta \boldsymbol{A}_{i-1}\right)\right\|_{L^{2}\left(\Omega_{c}\right)}^{2} \leq C
$$

Therefore,

$$
\max _{1 \leq j \leq n}\left\{\left\|\delta \boldsymbol{A}_{j}\right\|_{\boldsymbol{H}^{1}\left(\Omega_{c}\right)}^{2}+\left\|\delta \phi_{j}\right\|_{H^{1}\left(\Omega_{c}\right)}^{2}\right\}+\sum_{i=1}^{n}\left\|\delta \boldsymbol{A}_{i}-\delta \boldsymbol{A}_{i-1}\right\|_{\boldsymbol{H}^{1}\left(\Omega_{c}\right)}^{2} \leq C .
$$

Proof. We take the scalar product of (3.7) on $\Omega_{c}$ with $\nabla \times \delta \boldsymbol{A}_{i}$ and integrating over $\Omega_{c}$. Afterwards, we multiply the result by $\tau$ and sum up for $i=1, \cdots, j$ to obtain that

$$
\begin{aligned}
\sum_{i=1}^{j} \tau \varepsilon_{c}\left(\nabla \times \delta^{2} \boldsymbol{A}_{i}, \nabla \times \delta \boldsymbol{A}_{i}\right)_{\Omega_{c}}+\sum_{i=1}^{j} \tau & \left\|\nabla \times \delta \boldsymbol{A}_{i}\right\|_{\Omega_{c}}^{2} \\
& +\sum_{i=1}^{j} \frac{\tau}{\mu_{c}}\left(\nabla \times \nabla \times \nabla \times \boldsymbol{A}_{i}, \nabla \times \delta \boldsymbol{A}_{i}\right)_{\Omega_{c}}=\sum_{i=1}^{j} \tau\left(h_{i} \nabla \times \boldsymbol{f}, \nabla \times \delta \boldsymbol{A}_{i}\right)_{\Omega_{c}} .
\end{aligned}
$$

Abel's summation rule gives that

$$
\sum_{i=1}^{j} \tau \varepsilon_{c}\left(\nabla \times \delta^{2} \boldsymbol{A}_{i}, \nabla \times \delta \boldsymbol{A}_{i}\right)_{\Omega_{c}}=\frac{\varepsilon_{c}}{2}\left(\left\|\nabla \times \delta \boldsymbol{A}_{j}\right\|_{L^{2}\left(\Omega_{c}\right)}^{2}-\left\|\nabla \times \boldsymbol{A}_{0}^{\prime}\right\|_{L^{2}\left(\Omega_{c}\right)}^{2}+\sum_{i=1}^{j}\left\|\nabla \times\left(\delta \boldsymbol{A}_{i}-\delta \boldsymbol{A}_{i-1}\right)\right\|_{\boldsymbol{L}^{2}\left(\Omega_{c}\right)}^{2}\right) .
$$

Thanks to Theorem 3.5, we have that

$$
\left|\sum_{i=1}^{j} \frac{\tau}{\mu_{c}}\left(\nabla \times \nabla \times \nabla \times \boldsymbol{A}_{i}, \nabla \times \delta \boldsymbol{A}_{i}\right)_{\Omega_{c}}\right| \leq C_{\varepsilon}+\varepsilon \sum_{i=1}^{j}\left\|\nabla \times \delta \boldsymbol{A}_{i}\right\|_{L^{2}\left(\Omega_{c}\right)}^{2} \tau
$$

and

$$
\left|\sum_{i=1}^{j} \tau\left(h_{i} \nabla \times \boldsymbol{f}, \nabla \times \delta \boldsymbol{A}_{i}\right)_{\Omega_{c}}\right| \leq C_{\varepsilon}+\varepsilon \sum_{i=1}^{j}\left\|\nabla \times \delta \boldsymbol{A}_{i}\right\|_{\boldsymbol{L}^{2}\left(\Omega_{c}\right)}^{2} \tau .
$$

67 Fixing $\varepsilon$ sufficiently small concludes the proof.

Corollary 3.3. Let the assumptions of Theorem 3.5 be fulfilled. Then there exist positive constants $C$ and $\tau_{0}$ such that for all $0<\tau<\tau_{0}$ holds that

$$
\sum_{i=1}^{n}\left\|\delta^{2} \phi_{i}\right\|_{H^{1}(\Omega)}^{2} \tau+\sum_{i=1}^{n}\left\|\delta^{2} \boldsymbol{A}_{i}\right\|_{H^{1}(\Omega)}^{2} \tau \leq C .
$$

Proof. From equation (2.4) and Theorem 3.5 it follows that

$$
\sum_{i=1}^{n}\left\|\delta^{2}\left(\boldsymbol{A}_{i}+\nabla \phi_{i}\right)\right\|^{2} \tau \leq C
$$

From equation (3.7), Theorem 3.5 and Corollary 3.2 it follows that

$$
\sum_{i=1}^{n}\left\|\nabla \times \delta^{2} \boldsymbol{A}_{i}\right\|^{2} \tau=\sum_{i=1}^{n}\left\|\nabla \times \delta^{2} \boldsymbol{A}_{i}\right\|_{L^{2}\left(\Omega_{e}\right)}^{2} \tau+\sum_{i=1}^{n}\left\|\nabla \times \delta^{2} \boldsymbol{A}_{i}\right\|_{L^{2}\left(\Omega_{c}\right)}^{2} \tau \leq C .
$$

68 The result is a consequence of Lemma 2.1. 
Corollary 3.4. Let the assumptions of Theorem 3.5 be fulfilled. Then there exist positive constants $C$ and $\tau_{0}$ such that for all $0<\tau<\tau_{0}$ holds that

$$
\sum_{i=1}^{n}\left\|\delta \phi_{i}\right\|_{H^{1}(\Omega)}^{2} \tau+\sum_{i=1}^{n}\left\|\delta \boldsymbol{A}_{i}\right\|_{\boldsymbol{H}^{1}(\Omega)}^{2} \tau+\sum_{i=1}^{n}\left\|\delta \boldsymbol{A}_{i}-\delta \boldsymbol{A}_{i-1}\right\|_{\boldsymbol{H}^{1}(\Omega)}^{2}+\sum_{i=1}^{n}\left\|\delta \phi_{i}-\delta \phi_{i-1}\right\|_{H^{1}(\Omega)}^{2} \leq C .
$$

Proof. This result follows from

$$
\delta \phi_{j}=\phi_{0}^{\prime}+\sum_{i=1}^{j} \delta^{2} \phi_{i} \tau \quad \text { and } \quad \delta \boldsymbol{A}_{j}=\boldsymbol{A}_{0}^{\prime}+\sum_{i=1}^{j} \delta^{2} \boldsymbol{A}_{i} \tau
$$

and Corollary 3.3.

\section{Well-posedness}

Let us define some interpolations of the discretized fields in time by

$$
\begin{aligned}
& \left\{\begin{array}{l}
\boldsymbol{A}_{n}(t)=\boldsymbol{A}_{i-1}+\left(t-t_{i-1}\right) \delta \boldsymbol{A}_{i}, \quad t \in\left(t_{i-1}, t_{i}\right], \\
\boldsymbol{A}_{n}(0)=\boldsymbol{A}_{0},
\end{array}\right. \\
& \left\{\begin{array}{l}
\bar{A}_{n}(t)=\boldsymbol{A}_{i}, \quad t \in\left(t_{i-1}, t_{i}\right], \\
\bar{A}_{n}(0)=\boldsymbol{A}_{0},
\end{array}\right. \\
& \left\{\begin{array}{l}
\boldsymbol{V}_{n}(t)=\delta \boldsymbol{A}_{i-1}+\left(t-t_{i-1}\right) \delta^{2} \boldsymbol{A}_{i}, \quad t \in\left(t_{i-1}, t_{i}\right], \\
\boldsymbol{V}_{n}(0)=\boldsymbol{A}_{0}^{\prime},
\end{array}\right. \\
& \left\{\begin{array}{l}
\overline{\boldsymbol{V}}_{n}(t)=\delta \boldsymbol{A}_{i}, \quad t \in\left(t_{i-1}, t_{i}\right], \\
\overline{\boldsymbol{V}}_{n}(0)=\boldsymbol{A}_{0}^{\prime},
\end{array}\right. \\
& \left\{\begin{array}{l}
\phi_{n}(t)=\phi_{i-1}+\left(t-t_{i-1}\right) \delta \phi_{i}, \quad t \in\left(t_{i-1}, t_{i}\right], \\
\phi_{n}(0)=\phi_{0},
\end{array}\right. \\
& \left\{\begin{array}{l}
\bar{\phi}_{n}(t)=\phi_{i}, \quad t \in\left(t_{i-1}, t_{i}\right], \\
\bar{\phi}_{n}(0)=\phi_{0},
\end{array}\right. \\
& \left\{\begin{array}{l}
v_{n}(t)=\delta \phi_{i-1}+\left(t-t_{i-1}\right) \delta^{2} \phi_{i}, \quad t \in\left(t_{i-1}, t_{i}\right], \\
v_{n}(0)=\phi_{0}^{\prime},
\end{array}\right. \\
& \left\{\begin{array}{l}
\bar{v}_{n}(t)=\delta \phi_{i}, \quad t \in\left(t_{i-1}, t_{i}\right], \\
\bar{v}_{n}(0)=\phi_{0}^{\prime} .
\end{array}\right.
\end{aligned}
$$

Similarly, we define $\bar{h}_{n}, \bar{m}_{n}$ and ${\overline{m^{\prime}}}_{n}$. For $\left(\boldsymbol{A}_{0}, \phi_{0}\right)$ and $\left(\delta \boldsymbol{A}_{0}, \delta \phi_{0}\right)=\left(\boldsymbol{A}_{0}^{\prime}, \phi_{0}^{\prime}\right)$, the discretized problem (2.8)-(2.9) is rewritten as follows for all $(\boldsymbol{Q}, \psi) \in \boldsymbol{V}$ :

$$
\begin{aligned}
\left(\varepsilon \partial_{t}\left(\boldsymbol{V}_{n}(t)+\nabla v_{n}(t)\right), \boldsymbol{Q}+\nabla \psi\right)+\sigma_{c}\left(\partial _ { t } \left(\boldsymbol{A}_{n}(t)\right.\right. & \left.\left.+\nabla \phi_{n}(t)\right), \boldsymbol{Q}+\nabla \psi\right)_{\Omega_{c}} \\
& +\left(\frac{1}{\mu} \nabla \times \overline{\boldsymbol{A}}_{n}(t), \nabla \times \boldsymbol{Q}\right)+\left(\frac{1}{\mu} \nabla \cdot \overline{\boldsymbol{A}}_{n}(t), \nabla \cdot \boldsymbol{Q}\right)=\left(\bar{h}_{n}(t) \boldsymbol{f}, \boldsymbol{Q}+\nabla \psi\right),
\end{aligned}
$$

with

$$
\bar{h}_{n}(t)=\frac{-\varepsilon_{c}{\overline{m^{\prime}}}_{n}(t)-\sigma_{c} \bar{m}_{n}(t)+\frac{1}{\mu_{c}} \int_{\gamma} \nabla \times \nabla \times \overline{\boldsymbol{A}}_{n}(t-\tau) \cdot \boldsymbol{n} d \gamma}{\int_{\gamma} \boldsymbol{f} \cdot \boldsymbol{n} d \gamma} .
$$

The a priori estimates derived in the previous section can be written in the new notations as

$$
\int_{0}^{T} \bar{h}_{n}(t)^{2} d t \leq C
$$

and

$$
\begin{gathered}
\max _{t \in[0, T]}\left\{\left\|\overline{\boldsymbol{A}}_{n}(t)\right\|_{\boldsymbol{H}^{1}(\Omega)}^{2}+\left\|\nabla \times \nabla \times \overline{\boldsymbol{A}}_{n}(t)\right\|_{\boldsymbol{H}^{1}(\Omega)}^{2}+\left\|\partial_{t} \boldsymbol{A}_{n}(t)\right\|_{\boldsymbol{H}^{1}\left(\Omega_{c}\right)}^{2}+\left\|\nabla \times \nabla \times \partial_{t} \boldsymbol{A}_{n}(t)\right\|^{2}+\left\|\bar{\phi}_{n}(t)\right\|_{H^{1}(\Omega)}^{2}+\left\|\partial_{t} \phi_{n}(t)\right\|_{H^{1}\left(\Omega_{c}\right)}^{2}\right\} \\
+\int_{0}^{T}\left\|\partial_{t} \boldsymbol{A}_{n}(s)\right\|_{\boldsymbol{H}^{1}(\Omega)}^{2} d s+\int_{0}^{T}\left\|\partial_{t} \phi_{n}(s)\right\|_{H^{1}(\Omega)}^{2} d s+\int_{0}^{T}\left\|\partial_{t} \boldsymbol{V}_{n}(s)\right\|_{\boldsymbol{H}^{1}(\Omega)}^{2} d s \\
+\int_{0}^{T}\left\|\partial_{t} v_{n}(s)\right\|_{H^{1}(\Omega)}^{2} d s+\sum_{i=1}^{n}\left\|\int_{t_{i-1}}^{t_{i}} \partial_{t} \boldsymbol{A}_{n}\right\|_{\boldsymbol{H}^{1}(\Omega)}^{2}+\sum_{i=1}^{n}\left\|\int_{t_{i-1}}^{t_{i}} \partial_{t} \phi_{n}\right\|_{H^{1}(\Omega)}^{2}+\sum_{i=1}^{n}\left\|\nabla \times \nabla \times \int_{t_{i-1}}^{t_{i}} \partial_{t} \boldsymbol{A}_{n}\right\|_{\boldsymbol{H}^{1}(\Omega)}^{2} \\
+\sum_{i=1}^{n}\left\|\int_{t_{i-1}}^{t_{i}} \partial_{t} v_{n}\right\|_{H^{1}(\Omega)}^{2}+\sum_{i=1}^{n}\left\|\int_{t_{i-1}}^{t_{i}} \partial_{t} \boldsymbol{V}_{n}\right\|_{\boldsymbol{H}^{1}(\Omega)}^{2}+\sum_{i=1}^{n}\left\|\nabla \times \nabla \times \int_{t_{i-1}}^{t_{i}} \partial_{t} \boldsymbol{V}_{n}\right\|^{2} \leq C . \quad \text { (4.4) }
\end{gathered}
$$


Proof. Reflexivity of the space $L^{2}(0, T)$ together with $\int_{0}^{T} \bar{h}_{n}^{2}(t) d t \leq C$ gives for a subsequence (denoted by the same symbol again)

$$
\bar{h}_{n} \rightarrow z \text { in } L^{2}(0, T)
$$

The Rellich-Kondrachov theorem [25, p. 272] implies that

$$
\widehat{\boldsymbol{H}}_{0}^{1}(\Omega) \hookrightarrow \hookrightarrow \boldsymbol{L}^{2}(\Omega)
$$

It holds that (see equation (4.4))

$$
\max _{t \in[0, T]}\left\|\overline{\boldsymbol{A}}_{n}(t)\right\|_{\widehat{\boldsymbol{H}}_{0}^{1}(\Omega)}^{2}+\int_{0}^{T}\left\|\partial_{t} \boldsymbol{A}_{n}(s)\right\|^{2} d s \leq C,
$$

such that the conditions of [26, Lemma 1.3.13] are satisfied. Therefore, there exists $\boldsymbol{A} \in C\left([0, T], \boldsymbol{L}^{2}(\Omega)\right) \cap L^{\infty}\left((0, T), \widehat{\boldsymbol{H}}_{0}^{1}(\Omega)\right)$ and a subsequence $\left\{\boldsymbol{A}_{n_{k}}\right\}_{k \in \mathbb{N}}$ of $\left\{\boldsymbol{A}_{n}\right\}_{n \in \mathbb{N}}$ such that

$$
\left\{\begin{array}{lll}
\boldsymbol{A}_{n_{k}} \rightarrow \boldsymbol{A}, & \text { in } & C\left([0, T], \boldsymbol{L}^{2}(\Omega)\right), \\
\boldsymbol{A}_{n_{k}}(t) \rightarrow \boldsymbol{A}(t), & \text { in } \quad \widehat{\boldsymbol{H}}_{0}^{1}(\Omega) \text { for all } t \in[0, T], \\
\overline{\boldsymbol{A}}_{n_{k}}(t) \rightarrow \boldsymbol{A}(t), & \text { in } \widehat{\boldsymbol{H}}_{0}^{1}(\Omega) \text { for all } t \in[0, T], \\
\partial_{t} \boldsymbol{A}_{n_{k}}=\overline{\boldsymbol{V}}_{n_{k}} \rightarrow \partial_{t} \boldsymbol{A}, & \text { in } & L^{2}\left((0, T), \boldsymbol{L}^{2}(\Omega)\right) .
\end{array}\right.
$$

In addition, we have that $\nabla \cdot \overline{\boldsymbol{A}}_{n_{k}} \rightarrow \nabla \cdot \boldsymbol{A}$ in $L^{2}\left((0, T), \boldsymbol{L}^{2}(\Omega)\right)$. This follows from the density argument $\overline{C_{0}^{\infty}(\Omega)}=L^{2}(\Omega)$ and from the Green theorem because we obtain for all $\phi \in C_{0}^{\infty}(\Omega)$ that

$$
\begin{aligned}
\int_{0}^{\eta}\left(\nabla \cdot \overline{\boldsymbol{A}}_{n_{k}}(t), \phi\right) d t= & -\int_{0}^{\eta}\left(\overline{\boldsymbol{A}}_{n_{k}}(t), \nabla \phi\right) d t \\
& -\int_{0}^{\eta}(\boldsymbol{A}(t), \nabla \phi) d t \quad=\int_{0}^{\eta}(\nabla \cdot \boldsymbol{A}(t), \phi) d t .
\end{aligned}
$$

In an analogue way, there exist a function $\phi \in C\left([0, T], L^{2}(\Omega)\right) \cap L^{\infty}\left((0, T), H_{0}^{1}(\Omega)\right)$ and a subsequence $\left\{\phi_{n_{k}}\right\}_{k \in \mathbb{N}}$ of $\left\{\phi_{n}\right\}_{n \in \mathbb{N}}$ such that

$$
\left\{\begin{array}{lll}
\phi_{n_{k}} \rightarrow \phi, & \text { in } & C\left([0, T], L^{2}(\Omega)\right), \\
\phi_{n_{k}}(t) \rightarrow \phi(t), & \text { in } & H_{0}^{1}(\Omega) \text { for all } t \in[0, T], \\
\bar{\phi}_{n_{k}}(t) \rightarrow \phi(t), & \text { in } & H_{0}^{1}(\Omega) \text { for all } t \in[0, T], \\
\partial_{t} \phi_{n_{k}}=\bar{v}_{n_{k}} \rightarrow \partial_{t} \phi, & \text { in } & L^{2}\left((0, T), L^{2}(\Omega)\right) .
\end{array}\right.
$$

From $\int_{0}^{T}\left\|\partial_{t} \phi_{n}(s)\right\|_{H_{0}^{1}(\Omega)}^{2} d s \leq C$, it follows that

$$
\partial_{t} \nabla \phi_{n_{k}} \rightarrow \partial_{t} \nabla \phi \quad \text { in } L^{2}\left((0, T), \boldsymbol{L}^{2}(\Omega)\right)
$$

We denote these subsequences again with $\left\{\boldsymbol{A}_{n}\right\}$ and $\left\{\phi_{n}\right\}$ to skip double indices. From Corollary 3.4, it follows that

$$
\lim _{n \rightarrow \infty}\left\|\boldsymbol{V}_{n}-\overline{\boldsymbol{V}}_{n}\right\|_{L^{2}\left((0, T), \widehat{\boldsymbol{H}}_{0}^{1}(\Omega)\right)}^{2}=0=\lim _{n \rightarrow \infty}\left\|v_{n}-\bar{v}_{n}\right\|_{L^{2}\left((0, T), H_{0}^{1}(\Omega)\right)}^{2}
$$

Thus $\boldsymbol{V}_{n} \rightarrow \partial_{t} \boldsymbol{A}$ in $L^{2}\left((0, T), \widehat{\boldsymbol{H}}_{0}^{1}(\Omega)\right)$ and $v_{n} \rightarrow \partial_{t} \phi$ in $L^{2}\left((0, T), H_{0}^{1}(\Omega)\right)$. Moreover, thanks to

$$
\int_{0}^{T}\left\|\partial_{t} \boldsymbol{V}_{n}(s)\right\|_{\widehat{\boldsymbol{H}}_{0}^{1}(\Omega)}^{2} d s+\int_{0}^{T}\left\|\partial_{t} v_{n}(s)\right\|_{H_{0}^{1}(\Omega)}^{2} d s \leq C,
$$


we have that $\partial_{t} \boldsymbol{V}_{n} \rightarrow \partial_{t t} \boldsymbol{A}$ in $L^{2}\left((0, T), \widehat{\boldsymbol{H}}_{0}^{1}(\Omega)\right)$ and $\partial_{t} v_{n} \rightarrow \partial_{t t} \phi$ in $L^{2}\left((0, T), H_{0}^{1}(\Omega)\right)$. Now, we integrate (4.1) into time (for any $\xi \in(0, T)$ ) to get

$$
\begin{aligned}
\int_{0}^{\xi}\left(\varepsilon \partial_{t}\left(\boldsymbol{V}_{n}(t)+\nabla v_{n}(t)\right), \boldsymbol{Q}+\nabla \psi\right) & +\sigma_{c} \int_{0}^{\xi}\left(\partial_{t}\left(\boldsymbol{A}_{n}(t)+\nabla \phi_{n}(t)\right), \boldsymbol{Q}+\nabla \psi\right)_{\Omega_{c}} \\
& +\int_{0}^{\xi}\left(\frac{1}{\mu} \nabla \times \overline{\boldsymbol{A}}_{n}(t), \nabla \times \boldsymbol{Q}\right)+\int_{0}^{\xi}\left(\frac{1}{\mu} \nabla \cdot \overline{\boldsymbol{A}}_{n}(t), \nabla \cdot \boldsymbol{Q}\right)=\int_{0}^{\xi}\left(\bar{h}_{n}(t) \boldsymbol{f}, \boldsymbol{Q}+\nabla \psi\right) .
\end{aligned}
$$

Due to the convergence results above, we can pass to the limit $n \rightarrow \infty$ to obtain

$$
\begin{aligned}
\int_{0}^{\xi}\left(\varepsilon \partial_{t t}(\boldsymbol{A}(t)+\nabla \phi(t)), \boldsymbol{Q}+\nabla \psi\right)+\sigma_{c} & \int_{0}^{\xi}\left(\partial_{t}(\boldsymbol{A}(t)+\nabla \phi(t)), \boldsymbol{Q}+\nabla \psi\right)_{\Omega_{c}} \\
& +\int_{0}^{\xi}\left(\frac{1}{\mu} \nabla \times \boldsymbol{A}(t), \nabla \times \boldsymbol{Q}\right)+\int_{0}^{\xi}\left(\frac{1}{\mu} \nabla \cdot \boldsymbol{A}(t), \nabla \cdot \boldsymbol{Q}\right)=\int_{0}^{\xi}(z(t) \boldsymbol{f}, \boldsymbol{Q}+\nabla \psi) .
\end{aligned}
$$

Finally, we have to show that $z=h$. Afterwards, we can differentiate the last equality with respect to obtain the existence of a weak solution to (1.4)-(1.11).

To prove that $z=h$, i.e. the convergence of (4.2) to (2.1) as $n \rightarrow \infty$, we need better convergence of the approximates. From equation (4.4), it follows that

$$
\max _{t \in[0, T]}\left\{\left\|\nabla \times \nabla \times \overline{\boldsymbol{A}}_{n}(t)\right\|_{\widehat{\boldsymbol{H}}_{0}^{1}(\Omega)}^{2}+\left\|\nabla \times \nabla \times \partial_{t} \boldsymbol{A}_{n}(t)\right\|^{2}\right\} \leq C .
$$

Again, from Kačur's lemma, there exists $\nabla \times \nabla \times \boldsymbol{A} \in C\left([0, T], \boldsymbol{L}^{2}(\Omega)\right) \cap L^{\infty}\left((0, T), \boldsymbol{H}^{1}(\Omega)\right)$ and a subsequence $\{\nabla \times \nabla \times$ $\left.\boldsymbol{A}_{n_{k}}\right\}_{k \in \mathbb{N}}$ (denoted with the same symbol again) of $\left\{\nabla \times \nabla \times \boldsymbol{A}_{n}\right\}_{n \in \mathbb{N}}$ such that

$$
\left\{\begin{array}{lll}
\nabla \times \nabla \times \boldsymbol{A}_{n} \rightarrow \nabla \times \nabla \times \boldsymbol{A}, & \text { in } & C\left([0, T], \boldsymbol{L}^{2}(\Omega)\right), \\
\nabla \times \nabla \times \boldsymbol{A}_{n}(t)-\nabla \times \nabla \times \boldsymbol{A}(t), & \text { in } \quad \widehat{\boldsymbol{H}}_{0}^{1}(\Omega) \text { for all } t \in[0, T], \\
\nabla \times \nabla \times \overline{\boldsymbol{A}}_{n}(t)-\nabla \times \nabla \times \boldsymbol{A}(t), & \text { in } \quad \widehat{\boldsymbol{H}}_{0}^{1}(\Omega) \text { for all } t \in[0, T], \\
\partial_{t} \nabla \times \nabla \times \boldsymbol{A}_{n}=\nabla \times \nabla \times \overline{\boldsymbol{V}}_{n} \rightarrow \nabla \times \nabla \times \partial_{t} \boldsymbol{A}, & \text { in } \quad L^{2}\left((0, T), \boldsymbol{L}^{2}(\Omega)\right) .
\end{array}\right.
$$

Recalling the Nečas inequality

$$
\|z\|_{L^{2}(\partial G)}^{2} \leq \eta\|\nabla z\|_{L^{2}(G)}^{2}+C_{\eta}\|z\|_{L^{2}(G)}^{2}, \quad \forall z \in \boldsymbol{H}^{1}(G), \quad 0<\eta<\eta_{0},
$$

we have that

$$
\begin{aligned}
& \left|\int_{\gamma} \nabla \times \nabla \times\left(\boldsymbol{A}_{n}(t)-\boldsymbol{A}_{n}(t-\tau)\right) \cdot \boldsymbol{n} d \gamma\right|^{2} \\
& \leq C\left\|\nabla \times \nabla \times\left(\boldsymbol{A}_{n}(t)-\boldsymbol{A}_{n}(t-\tau)\right)\right\|_{\boldsymbol{L}^{2}(\gamma)}^{2} \\
& \stackrel{\gamma \subset G}{\leq} C\left\|\nabla \times \nabla \times\left(\boldsymbol{A}_{n}(t)-\boldsymbol{A}_{n}(t-\tau)\right)\right\|_{\boldsymbol{L}^{2}(\partial G)}^{2} \\
& \stackrel{(4.8)}{\leq} \eta\left\|\nabla \times \nabla \times\left(\boldsymbol{A}_{n}(t)-\boldsymbol{A}_{n}(t-\tau)\right)\right\|_{\boldsymbol{H}^{1}(G)}^{2}+C_{\eta}\left\|\nabla \times \nabla \times\left(\boldsymbol{A}_{n}(t)-\boldsymbol{A}_{n}(t-\tau)\right)\right\|_{L^{2}(G)}^{2} \\
& \stackrel{G \subset \Omega}{\leq} \eta\left\|\nabla \times \nabla \times\left(\boldsymbol{A}_{n}(t)-\boldsymbol{A}_{n}(t-\tau)\right)\right\|_{\boldsymbol{H}^{1}(\Omega)}^{2}+C_{\eta}\left\|\nabla \times \nabla \times\left(\boldsymbol{A}_{n}(t)-\boldsymbol{A}_{n}(t-\tau)\right)\right\|^{2} \\
& \leq \eta\left\|\nabla \times \nabla \times\left(\boldsymbol{A}_{n}(t)-\boldsymbol{A}_{n}(t-\tau)\right)\right\|_{\boldsymbol{H}^{1}(\Omega)}^{2}+C_{\eta} \int_{t-\tau}^{t}\left\|\partial_{t} \nabla \times \nabla \times \boldsymbol{A}_{n}(s)\right\|^{2} d s \\
& \leq \eta+C_{\eta} \tau \text {. }
\end{aligned}
$$

Passing to the limit for $n \rightarrow \infty$ and then for $\eta \rightarrow 0$, we find that

$$
\lim _{n \rightarrow \infty}\left|\int_{\gamma} \nabla \times \nabla \times\left(\boldsymbol{A}_{n}(t)-\boldsymbol{A}_{n}(t-\tau)\right) \cdot \boldsymbol{n} d \gamma\right|^{2}=0 .
$$


In a similar way, we deduce that

$$
\begin{aligned}
\left|\int_{\gamma} \nabla \times \nabla \times\left(\boldsymbol{A}_{n}(t)-\boldsymbol{A}(t)\right) \cdot \boldsymbol{n} d \gamma\right|^{2} & \leq C\left\|\nabla \times \nabla \times\left(\boldsymbol{A}_{n}(t)-\boldsymbol{A}(t)\right)\right\|_{\boldsymbol{L}^{2}(\gamma)}^{2} \\
& \leq C\left\|\nabla \times \nabla \times\left(\boldsymbol{A}_{n}(t)-\boldsymbol{A}(t)\right)\right\|_{\boldsymbol{L}^{2}(\partial G)}^{2} \\
& \leq \eta\left\|\nabla \times \nabla \times\left(\boldsymbol{A}_{n}(t)-\boldsymbol{A}(t)\right)\right\|_{\boldsymbol{H}^{1}(G)}^{2}+C_{\eta}\left\|\nabla \times \nabla \times\left(\boldsymbol{A}_{n}(t)-\boldsymbol{A}(t)\right)\right\|_{\boldsymbol{L}^{2}(G)}^{2} \\
& \leq \eta\left\|\nabla \times \nabla \times\left(\boldsymbol{A}_{n}(t)-\boldsymbol{A}(t)\right)\right\|_{\boldsymbol{H}^{1}(\Omega)}^{2}+C_{\eta}\left\|\nabla \times \nabla \times\left(\boldsymbol{A}_{n}(t)-\boldsymbol{A}(t)\right)\right\|^{2} \\
& \leq \eta+C_{\eta}\left\|\nabla \times \nabla \times\left(\boldsymbol{A}_{n}(t)-\boldsymbol{A}(t)\right)\right\|^{2} .
\end{aligned}
$$

Passing to the limit for $n \rightarrow \infty$ and using

$$
\nabla \times \nabla \times \boldsymbol{A}_{n} \rightarrow \nabla \times \nabla \times \boldsymbol{A} \text { for a.e. }(\boldsymbol{x}, t) \in \Omega \times[0, T],
$$

we get

$$
\lim _{n \rightarrow \infty}\left|\int_{\gamma} \nabla \times \nabla \times\left(\boldsymbol{A}_{n}(t-\tau)-\boldsymbol{A}(t)\right) \cdot \boldsymbol{n} d \gamma\right|^{2} \leq \eta .
$$

The value of $\eta>0$ can be taken arbitrarily small. Thus, we obtain that

$$
\lim _{n \rightarrow \infty}\left|\int_{\gamma} \nabla \times \nabla \times\left(\boldsymbol{A}_{n}(t-\tau)-\boldsymbol{A}(t)\right) \cdot \boldsymbol{n} d \gamma\right|^{2}=0
$$

Therefore, we conclude that

$$
\lim _{n \rightarrow \infty}\left|\int_{\gamma} \nabla \times \nabla \times\left(\overline{\boldsymbol{A}}_{n}(t-\tau)-\boldsymbol{A}(t)\right) \cdot \boldsymbol{n} d \gamma\right|^{2}=0 .
$$

Since the measurement $m \in C^{1}([0, T])$, we have that $\bar{m}_{n}(t) \rightarrow m(t)$ and ${\overline{m^{\prime}}}_{n}(t) \rightarrow m^{\prime}(t)$ for any $t \in[0, T]$. Collecting the considerations above we see that we may pass to the limit for $n \rightarrow \infty$ in (4.2) to arrive at (2.3), i.e. $z=h$. Note that for the equation system (2.4)

$$
\begin{cases}\varepsilon \partial_{t}\left(\boldsymbol{V}_{n}+\nabla v_{n}\right)+\sigma \partial_{t}\left(\boldsymbol{A}_{n}+\nabla \phi_{n}\right)+\nabla \times\left(\frac{1}{\mu} \nabla \times \overline{\boldsymbol{A}}_{n}\right)-\nabla\left(\frac{1}{\mu} \nabla \cdot \overline{\boldsymbol{A}}_{n}\right)=\bar{h}_{n} \boldsymbol{f} & \text { in } \Omega \times(0, T], \\ \nabla \cdot\left(\varepsilon \partial_{t t}\left(\boldsymbol{A}_{n}+\nabla \phi_{n}\right)+\sigma \partial_{t}\left(\boldsymbol{A}_{n}+\nabla \phi_{n}\right)\right)=\bar{h}_{n} \nabla \cdot \boldsymbol{f} & \text { in } \Omega \times(0, T],\end{cases}
$$

and the equation system (1.4)

$$
\begin{cases}\varepsilon \partial_{t t}(\boldsymbol{A}+\nabla \phi)+\sigma \partial_{t}(\boldsymbol{A}+\nabla \phi)+\nabla \times\left(\frac{1}{\mu} \nabla \times \boldsymbol{A}\right)-\nabla\left(\frac{1}{\mu} \nabla \cdot \boldsymbol{A}\right)=h \boldsymbol{f} & \text { in } \Omega \times(0, T], \\ \nabla \cdot\left(\varepsilon \partial_{t t}(\boldsymbol{A}+\nabla \phi)+\sigma \partial_{t}(\boldsymbol{A}+\nabla \phi)\right)=h \nabla \cdot \boldsymbol{f} & \text { in } \Omega \times(0, T],\end{cases}
$$

taking divergence to both side of the first equations of the two systems respectively and considering proper boundary conditions, we conclude that

$$
\nabla \cdot \overline{\boldsymbol{A}}_{n}(t)=0, \quad \nabla \cdot \boldsymbol{A}(t)=0, \quad \forall t \in(0, T]
$$

Finally, we discuss the uniqueness of a solution to the inverse problem (1.4)-(1.11).

Theorem 4.2. Let the assumptions of Theorem 4.1 be satisfied. Then, there exists a unique weak solution to (1.4)-(1.11).

Proof. Due to the linearity of the problem it is sufficient to show that if $m=0$ and $\left(\boldsymbol{A}_{0}, \phi_{0}\right)=(\mathbf{0}, 0)$ and $\left(\boldsymbol{A}_{0}^{\prime}, \phi_{0}^{\prime}\right)=(\mathbf{0}, 0)$ then $(\boldsymbol{A}(t), \phi(t), h(t))=(\mathbf{0}, 0,0)$ for any $t \in[0, T]$. The proof is split into several steps.

1st step: We integrate the system (1.4) over the time variable $t \in(0, s) \subset(0, T)$ to get

$$
\varepsilon \partial_{t}(\boldsymbol{A}(s)+\nabla \phi(s))+\sigma(\boldsymbol{A}(s)+\nabla \phi(s))+\int_{0}^{s} \nabla \times\left(\frac{1}{\mu} \nabla \times \boldsymbol{A}\right)=\int_{0}^{s} h \boldsymbol{f},
$$




$$
\nabla \cdot\left(\varepsilon \partial_{t}(\boldsymbol{A}(s)+\nabla \phi(s))+\sigma(\boldsymbol{A}(s)+\nabla \phi(s))\right)=\int_{0}^{s} h \nabla \cdot \boldsymbol{f} .
$$

We take the scalar product of the above two equations with $\boldsymbol{A}(s)$ and $\phi(s)$ respectively, integrate over $\Omega$, make use of the Green theorem and sum them together to obtain

$$
\left(\varepsilon \partial_{t}(\boldsymbol{A}(s)+\nabla \phi(s)), \boldsymbol{A}(s)+\nabla \phi(s)\right)+(\sigma(\boldsymbol{A}(s)+\nabla \phi(s)), \boldsymbol{A}(s)+\nabla \phi(s))+\left(\int_{0}^{s} \frac{1}{\mu} \nabla \times \boldsymbol{A}, \nabla \times \boldsymbol{A}(s)\right)=\left(\int_{0}^{s} h \boldsymbol{f}, \boldsymbol{A}(s)+\nabla \phi(s)\right) .
$$

Integrating in time over the time variable $s \in(0, t) \subset(0, T)$ yields

$$
\frac{\varepsilon_{\min }}{2}\|\boldsymbol{A}(t)+\nabla \phi(t)\|^{2}+\sigma_{c} \int_{0}^{t}\|\boldsymbol{A}(s)+\nabla \phi(s)\|_{\boldsymbol{L}^{2}\left(\Omega_{c}\right)}^{2} d s+\int_{0}^{t}\left(\int_{0}^{s} \frac{1}{\mu} \nabla \times \boldsymbol{A}, \nabla \times \boldsymbol{A}(s)\right) d s=\int_{0}^{t}\left(\int_{0}^{s} h \boldsymbol{f}, \boldsymbol{A}(s)+\nabla \phi(s)\right) d s .
$$

For the term on the RHS, we have that

$$
\left|\int_{0}^{t}\left(\int_{0}^{s} h \boldsymbol{f}, \boldsymbol{A}(s)+\nabla \phi(s)\right) d s\right| \leq C \int_{0}^{t}\|\boldsymbol{A}(s)+\nabla \phi(s)\|^{2} d s+C \int_{0}^{t} h^{2}(s) d s .
$$

We obtain for the third term on the LHS that

$$
\begin{aligned}
\int_{0}^{t}\left(\int_{0}^{s} \frac{1}{\mu} \nabla \times \boldsymbol{A}, \nabla \times \boldsymbol{A}(s)\right) d s & =\int_{0}^{t}\left(\int_{0}^{s} \frac{1}{\mu_{c}} \nabla \times \boldsymbol{A}, \nabla \times \boldsymbol{A}(s)\right)_{\Omega_{c}} d s+\int_{0}^{t}\left(\int_{0}^{s} \frac{1}{\mu_{e}} \nabla \times \boldsymbol{A}, \nabla \times \boldsymbol{A}(s)\right)_{\Omega_{e}} d s \\
& =\frac{1}{2 \mu_{c}}\left\|\int_{0}^{t} \nabla \times \boldsymbol{A}\right\|_{L^{2}\left(\Omega_{c}\right)}^{2}+\frac{1}{2 \mu_{e}}\left\|\int_{0}^{t} \nabla \times \boldsymbol{A}\right\|_{\boldsymbol{L}^{2}\left(\Omega_{e}\right)}^{2} \\
& \geq \min \left\{\frac{1}{2 \mu_{c}}, \frac{1}{2 \mu_{e}}\right\}\left\|\int_{0}^{t} \nabla \times \boldsymbol{A}\right\|^{2} .
\end{aligned}
$$

The second term in the LHS gives no better information and can be omitted. Applying the Grönwall inequality, we conclude that

$$
\|\boldsymbol{A}(t)+\nabla \phi(t)\|^{2}+\left\|\int_{0}^{t} \nabla \times \boldsymbol{A}\right\|^{2} \leq C \int_{0}^{t} h^{2}(s) d s, \quad \forall t \in[0, T] .
$$

2nd step: We put $\boldsymbol{Q}=\partial_{t} \boldsymbol{A}(t)$ and $\psi=\partial_{t} \phi(t)$ in (2.3). Then, we integrate in time and estimate the resulting RHS. We obtain that

$$
\frac{\varepsilon_{\min }}{2}\left\|\partial_{t}(\boldsymbol{A}(t)+\nabla \phi(t))\right\|^{2}+\sigma_{c} \int_{0}^{t}\left\|\partial_{t}(\boldsymbol{A}+\nabla \phi)\right\|_{L^{2}\left(\Omega_{c}\right)}^{2}+\frac{1}{2 \mu_{\max }}\|\nabla \times \boldsymbol{A}(t)\|^{2} \leq C \int_{0}^{t}\left\|\partial_{t}(\boldsymbol{A}+\nabla \phi)\right\|^{2}+C \int_{0}^{t} h^{2}(s) d s .
$$

Applying the Grönwall inequality, we conclude that

$$
\left\|\partial_{t}(\boldsymbol{A}(t)+\nabla \phi(t))\right\|^{2}+\|\nabla \times \boldsymbol{A}(t)\|^{2} \leq C \int_{0}^{t} h^{2}(s) d s, \quad \forall t \in[0, T] .
$$

3rd step: From equation (1.4), it follows that

$$
\begin{aligned}
\nabla \times\left(\frac{1}{\mu} \nabla \times \boldsymbol{A}\right) \times \boldsymbol{n} & =h \boldsymbol{f} \times \boldsymbol{n}-\varepsilon \partial_{t t} \boldsymbol{A} \times \boldsymbol{n}-\sigma \partial_{t} \boldsymbol{A} \times \boldsymbol{n}=\mathbf{0} \quad \text { on } \partial \Omega, \\
{\left[\nabla \times\left(\frac{1}{\mu} \nabla \times \boldsymbol{A}\right) \times \boldsymbol{n}_{12}\right] } & =\mathbf{0} \quad \text { on } \Gamma,
\end{aligned}
$$

Applying the curl operator to the first equation of (1.4) gives

$$
\nabla \times\left(\varepsilon \partial_{t t} \boldsymbol{A}\right)+\nabla \times\left(\sigma \partial_{t} \boldsymbol{A}\right)+\nabla \times \nabla \times\left(\frac{1}{\mu} \nabla \times \boldsymbol{A}\right)=h \nabla \times f .
$$

Integrating in time over $(0, s)$, we obtain that

$$
\nabla \times\left(\varepsilon \partial_{t} \boldsymbol{A}(s)\right)+\nabla \times(\sigma \boldsymbol{A}(s))+\int_{0}^{s} \nabla \times \nabla \times\left(\frac{1}{\mu} \nabla \times \boldsymbol{A}\right)=\int_{0}^{s} h \nabla \times \boldsymbol{f}
$$


Now, we take a scalar product with $\nabla \times \nabla \times\left(\frac{1}{\mu} \nabla \times \boldsymbol{A}(s)\right)$, integrate over $\Omega$ and make use of the Green theorem to get

$$
\begin{aligned}
\left(\nabla \times \nabla \times\left(\varepsilon \partial_{t} \boldsymbol{A}(s)\right), \nabla \times\right. & \left.\times\left(\frac{1}{\mu} \nabla \times \boldsymbol{A}(s)\right)\right)+\left(\nabla \times \nabla \times(\sigma \boldsymbol{A}(s)), \nabla \times\left(\frac{1}{\mu} \nabla \times \boldsymbol{A}(s)\right)\right) \\
& +\left(\int_{0}^{s} \nabla \times \nabla \times\left(\frac{1}{\mu} \nabla \times \boldsymbol{A}\right), \nabla \times \nabla \times\left(\frac{1}{\mu} \nabla \times \boldsymbol{A}(s)\right)\right)=\left(\int_{0}^{s} h \nabla \times \nabla \times f, \nabla \times\left(\frac{1}{\mu} \nabla \times \boldsymbol{A}(s)\right)\right) .
\end{aligned}
$$

Integrating in time again leads to

$$
\begin{aligned}
& \|\nabla \times \nabla \times \boldsymbol{A}(t)\|^{2}+\int_{0}^{t}\|\nabla \times \nabla \times \boldsymbol{A}(s)\|_{L^{2}\left(\Omega_{c}\right)}^{2} d s+\left\|\int_{0}^{t} \nabla \times \nabla \times \nabla \times \boldsymbol{A}\right\|^{2} \\
& \leq C \int_{0}^{t}\left\|\int_{0}^{s} h \nabla \times \nabla \times f\right\|^{2} d s+C \int_{0}^{t}\left\|\nabla \times\left(\frac{1}{\mu} \nabla \times \boldsymbol{A}(s)\right)\right\|^{2} d s \\
& \leq C \int_{0}^{t} h^{2}(s) d s+C \int_{0}^{t}\|\nabla \times \nabla \times \boldsymbol{A}(s)\|^{2} d s .
\end{aligned}
$$

Applying the Grönwall inequality yields

$$
\|\nabla \times \nabla \times \boldsymbol{A}(t)\|^{2}+\left\|\int_{0}^{t} \nabla \times \nabla \times \nabla \times \boldsymbol{A}\right\|^{2} \leq C \int_{0}^{t} h^{2}(s) d s, \quad \forall t \in[0, T] .
$$

4th step: We take the scalar product of equation (4.10) with $\nabla \times \nabla \times\left(\frac{1}{\mu} \nabla \times \partial_{t} \boldsymbol{A}(t)\right)$, integrate over $\Omega$, make use of the Green theorem and integrate in time to get

$$
\left\|\nabla \times \nabla \times \partial_{t} \boldsymbol{A}(t)\right\|^{2}+\int_{0}^{t}\left\|\nabla \times \nabla \times \partial_{t} \boldsymbol{A}(s)\right\|_{\mathbf{L}^{2}\left(\Omega_{c}\right)}^{2} d s+\|\nabla \times \nabla \times \nabla \times \boldsymbol{A}(t)\|^{2} \leq C \int_{0}^{t} h^{2}(s) d s+C \int_{0}^{t}\left\|\nabla \times \nabla \times \partial_{t} \boldsymbol{A}(s)\right\|^{2} d s .
$$

Again, an application of Grönwall's inequality gives

$$
\left\|\nabla \times \nabla \times \partial_{t} \boldsymbol{A}(t)\right\|^{2}+\|\nabla \times \nabla \times \nabla \times \boldsymbol{A}(t)\|^{2} \leq C \int_{0}^{t} h^{2}(s) d s, \quad \forall t \in[0, T] .
$$

5th step: We start from (2.3) with $m=0$ to deduce that

$$
\begin{aligned}
& \int_{0}^{t} h^{2}(s) d s \quad=\quad \int_{0}^{t}\left|\frac{\frac{1}{\mu_{c}} \int_{\gamma} \nabla \times \nabla \times \boldsymbol{A}(s) \cdot \boldsymbol{n} d \gamma}{\int_{\gamma} \boldsymbol{f} \cdot \boldsymbol{n} d \gamma}\right|^{2} d s \\
& \leq \quad C \int_{0}^{t}\left(\int_{\gamma}|\nabla \times \nabla \times \boldsymbol{A}(s) \cdot \boldsymbol{n}|^{2} d \gamma\right) d s \\
& \leq \quad C \int_{0}^{t}\|\nabla \times \nabla \times A(s)\|_{L^{2}(\gamma)}^{2} d s
\end{aligned}
$$

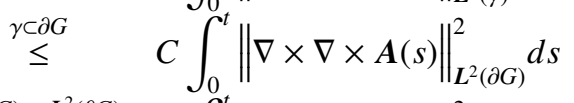

$$
\begin{aligned}
& \stackrel{\boldsymbol{H}^{1}(G) \hookrightarrow L^{2}(\partial G)}{\leq} C \int_{0}^{t}\|\nabla \times \nabla \times A(s)\|_{\boldsymbol{H}^{1}(G)}^{2} d s \\
& \stackrel{G \subset \Omega}{\leq} \quad C \int_{0}^{t}\|\nabla \times \nabla \times \nabla \times \boldsymbol{A}(s)\|^{2} d s+C \int_{0}^{t}\|\nabla \times \nabla \times \boldsymbol{A}(s)\|^{2} d s .
\end{aligned}
$$

From step 3 and 4 follows for all $t \in[0, T]$ that

$$
\int_{0}^{t} h^{2}(s) d s \leq \int_{0}^{t} \int_{0}^{s} h^{2}(\xi) d \xi d s
$$

Therefore, by Grönwall's argument, it holds that

$$
\int_{0}^{T} h^{2}(s) d s=0 \quad \text { or } \quad h=0 \text { in } L^{2}(0, T) .
$$

Moreover, step 1 and 2 imply that

$$
\max _{t \in[0, T]}\|A(t)+\nabla \phi(t)\|^{2}+\max _{\substack{t \in[0, T] \\ 19}}\left\|\int_{0}^{t} \nabla \times A\right\|^{2}=0
$$


and

$$
\max _{t \in[0, T]}\left\|\partial_{t}(\boldsymbol{A}(t)+\nabla \phi(t))\right\|^{2}+\max _{t \in[0, T]}\|\nabla \times \boldsymbol{A}(t)\|^{2}=0 .
$$

Then, from Lemma 2.1, it follows that

$$
\max _{t \in[0, T]}\|\boldsymbol{A}(t)\|_{\boldsymbol{H}^{1}(\Omega)}^{2}+\max _{t \in[0, T]}\|\phi(t)\|_{H^{1}(\Omega)}^{2}=0,
$$

which concludes the proof of uniqueness.

Corollary 4.1. Let the assumptions of Theorem 4.1 be satisfied. Then, there exists a unique solution $(\boldsymbol{E}, \boldsymbol{H}, h)$ to $(1.1)$, (1.2) and (1.11) satisfying $\boldsymbol{E}, \boldsymbol{H} \in C\left([0, T], \boldsymbol{L}^{2}(\Omega)\right)$ and $h \in L^{2}(0, T)$.

Remark 4.1. Theorem 3.1 shows the convergence of the Rothe functions for a subsequence. Theorem 3.2 ensures the uniqueness of a solution. Combination of both results implies convergence for the whole sequences of approximation $\boldsymbol{A}_{n}$, $\phi_{n}$ and $h_{n}$.

Remark 4.2. The analysis presented in this paper stays valid when instead one subdomain $\Omega_{c}$ multiple subdomains $\Omega_{c_{i}}$ are considered (where $\sigma, \varepsilon$ and $\mu$ are piecewise constant). Moreover, the analysis stays valid when the functions are not piecewise constant. Then, the following vector derivative identities need to be used

$$
\begin{aligned}
\nabla \times(\phi \boldsymbol{A}) & =\phi \nabla \times \boldsymbol{A}+\nabla \phi \times \boldsymbol{A}, \\
\nabla \times(\boldsymbol{A} \times \boldsymbol{B}) & =(\boldsymbol{B} \cdot \nabla) \boldsymbol{A}-(\boldsymbol{A} \cdot \nabla) \boldsymbol{B}+\boldsymbol{A}(\nabla \cdot \boldsymbol{B})-\boldsymbol{B}(\nabla \cdot \boldsymbol{A}) .
\end{aligned}
$$

For instance, the terms $\nabla \times\left(\frac{1}{\mu} \nabla \times \boldsymbol{A}\right), \nabla \times \nabla \times\left(\varepsilon \partial_{t t} \boldsymbol{A}\right)$ and $\nabla \times \nabla \times\left(\sigma \partial_{t} \boldsymbol{A}\right)$ need to be handled. In that case, the functions $\sigma, \varepsilon$ and $\mu$ need to be piecewise twice differentiable.

\section{Numerical experiments}

In the following, multiple numerical experiments are considered, each in a different setting but with the same exact solution on the whole domain $\Omega=(0,1)^{3}$ given by

$$
\boldsymbol{A}_{\mathrm{ex}}(\boldsymbol{x}, t)=\exp (t)(\sin y, \sin z, \sin x), \quad \phi_{\mathrm{ex}}(\boldsymbol{x}, t)=-\exp (t)(\cos x+\cos y+\cos z) \quad \text { and } \quad h_{\mathrm{ex}}(t)=\exp (t) .
$$

Then, the RHS of (1.4) equals

$$
h \boldsymbol{f}=h\left(\begin{array}{c}
(\varepsilon+\sigma) \sin x+\left(\varepsilon+\sigma+\frac{1}{\mu}\right) \sin y \\
(\varepsilon+\sigma) \sin y+\left(\varepsilon+\sigma+\frac{1}{\mu}\right) \sin z \\
(\varepsilon+\sigma) \sin z+\left(\varepsilon+\sigma+\frac{1}{\mu}\right) \sin x
\end{array}\right) .
$$

In Experiment 1, it is assumed that

$$
\Omega_{c}=\Omega \text { with } \gamma=\left\{(x, y, z) \in \partial \Omega_{c}=\partial \Omega: x=0, y \in(0.2,0.6), z \in(0.4,0.6)\right\}, \mu=\sigma=\varepsilon=1,
$$

whilst in Experiment 2 it holds that

$$
\Omega_{c}=\Omega \text { with } \gamma=\left\{(x, y, z) \in \Omega_{c}: x=0.4, y \in(0.2,0.4), z \in(0.4,0.6)\right\}, \mu=\sigma=\varepsilon=1,
$$

and in the challenging Experiment 3, it is assumed that

$$
\Omega_{c}=(0.4,0.6)^{3} \subset \Omega \text { with } \gamma=\left\{(x, y, z) \in \partial \Omega_{c}: x \in(0.4,0.6), y=0.4, z \in(0.4,0.6)\right\}, \sigma_{c}=1, \mu=\varepsilon= \begin{cases}1 & \text { in } \bar{\Omega}_{c}, \\ 0.5 & \text { in } \Omega \backslash \bar{\Omega}_{c} .\end{cases}
$$

Thus in Experiment 1, the measurement is on the surface of the conductor, whilst in Experiment 2, the measurement is on a surface inside the conductor. In Experiment 3, the measurement is again on the surface of the conductor but now the conductor is part of a larger domain and the coefficients are discontinuous. In this latter experiment, the interface conditions need to be handled carefully because the magnetic permeability is discontinuous (but piecewise constant). 
First, the variational formulation on $\Omega_{c}$ and $\Omega_{e}$ is mutiplied with the corresponding magnetic permeability. Then, the resulting formulations are added together to obtain for all $(\boldsymbol{Q}, \psi) \in \boldsymbol{V}$ that

$$
\begin{array}{r}
\left(\mu \varepsilon \partial_{t t}(\boldsymbol{A}(t)+\nabla \phi(t)), \boldsymbol{Q}+\nabla \psi\right)+\left(\mu \sigma \partial_{t}(\boldsymbol{A}(t)+\nabla \phi(t)), \boldsymbol{Q}+\nabla \psi\right)+(\nabla \times \boldsymbol{A}(t), \nabla \times \boldsymbol{Q})+(\nabla \cdot \boldsymbol{A}(t), \nabla \cdot \boldsymbol{Q}) \\
-\left(\left[\boldsymbol{n}_{12} \times(\nabla \times \boldsymbol{A}(t))\right], \boldsymbol{Q}\right)_{\partial \Omega_{c}}+\left([\nabla \cdot \boldsymbol{A}(t)], \boldsymbol{Q} \cdot \boldsymbol{n}_{12}\right)_{\partial \Omega_{c}}-\left(\left[\nabla \times \nabla \times \boldsymbol{A}(t) \cdot \boldsymbol{n}_{12}-\nabla(\nabla \cdot \boldsymbol{A}(t)) \cdot \boldsymbol{n}_{12}\right], \psi\right)_{\partial \Omega_{c}} \\
=(\mu h(t) \boldsymbol{f}, \boldsymbol{Q}+\nabla \psi) .
\end{array}
$$

Finally, thanks to the choice of the exact solution, the interface integrals cancel out.

The exact measurement (1.11) in the different experiments is given by

$$
m(t)=0.03094619258 \exp (t),-0.02737785046 \exp (t) \text { and } 0.03472180952 \exp (t),
$$

respectively. A randomly generated uncorrelated noise with magnitude $\tilde{e}$ is added to the exact measurement in order to simulate the inherent errors present in real measurements. The algorithm proposed in this contribution uses first order derivatives of the measurement, which enlarges the noise when using for instance finite difference to approximate the derivatives of noisy data. For this reason, the nonlinear least square method is used to get a sufficiently smooth function $m_{\mathrm{app}, \tilde{e}}(t)=\alpha \exp (\beta t)$ approximating the noisy measurement. Thus, the main goal of the experiments is to recover $h(t)$ by means of the measurement $m_{\mathrm{app}, \tilde{e}}(t)$ in the different settings.

The solution to the inverse source problems is found by applying the algorithm proposed in Section 2 . The problem (1.4) is discretized in time according to the backward Euler method. In each experiment, it is assumed that $T=1$. The time step for the equidistant time partitioning equals 0.005 in Experiment 1 and 0.0025 in Experiment 2 and 3 . At each time step, first $h_{i}$ is derived by numerical integration (see (2.5)), then the resulting elliptic problem (2.4) is solved numerically by the finite element method using second order (P2-FEM) Lagrange finite elements for the space discretization (for both $\boldsymbol{A}_{i}$ and $\left.\phi_{i}\right)$. An uniform mesh for the space domain is created consisting out of $6000(=6 \times 10 \times 10 \times 10)$ tetrahedra. For these calculations, the finite element library DOLFIN [28, 29] from the FEniCS project [30, 31] is used. The MUMPS solver is used to solve the linear systems at each timestep in order to speed up the computational process.

The numerical results for different noise levels $\tilde{e}=1 \%, 5 \%$ and $10 \%$ are depicted in Figure 1. In Figures 1(a,c,e), the numerical solution is compared with the exact solution. Moreover, in Figures 1(b,d,f), the absolute error on $\boldsymbol{E}$ at final time $T=1$ is presented for each experiment using $\tilde{e}=5 \%$. The CPU time (in minutes) and computational errors for the experiments using $\tilde{e}=0 \%$ can be found in Table 1 . The CPU time is comparable for the similar experiments with noise included. The maximum error on $h$ for all experiments is given in Table 2. It can be seen that, as expected, the error increases when the amount of noise increases. Moreover, it is clear from the figures that an accurate approximation of the source is obtained for each experiment (and each noise level). These experiments illustrate the robustness of the numerical procedure.

Remark 5.1. The use of nodal elements comes from regularizing Equation 1.4 by adding the penalty term. However, the use of nodal finite elements is not state of the art in case of domains with reentrant corners at the boundary (this is excluded by the assumption that the domain is convex) or in case of corners at material interfaces [32]. Also [33] pointed out that application of the linear tetrahedral element to vector problems is problematic. Because of numerical error, the gauge condition is not satisfied exactly. If there exists media having vastly different $\mu$, a small value of $\nabla \cdot \boldsymbol{A}$ in a medium having a low $\mu$ could lead to a very large value of $\nabla \cdot \boldsymbol{A}$ in a medium having a high $\mu$, As a consequence, it is impossible for $\boldsymbol{A}$ to satisfy the original problem reasonably, particularly in a medium having a high $\mu$. In all experiments quadratic tetrahedral elements are used (these elements are also necessary for the calculation of $h$ at each timestep) and moreover the variational formulation (5.1) is used to reduce this problem. This latter remark is tested in Experiment 4 wherein Experiment 3 is repeated with higher contrast in the parameters, i.e $\sigma_{c}=\mu_{c}=\varepsilon_{c}=1000$. The results are depicted in Figure 2. The absolute error on $\boldsymbol{A}$ and $\boldsymbol{B}$ at final time is good but the absolute error on $\boldsymbol{E}$ is less good in comparison with the previous experiments, which is clearly visible at the corners of the conductor. Nevertheless, the results obtained for the recovery of $h$ are fine, which is the main aim of this paper.

\section{Conclusion}

In this paper, an inverse source problem associated with full Maxwell's equations was considered. More precisely, the theoretical and numerical determination of a time-dependent source in a hyperbolic partial differential equation in terms of the electric field and magnetic field was studied from the knowledge of a surface measurement. An algorithm based on the $\boldsymbol{A}-\boldsymbol{\phi}$ method and on Rothe's method was proposed for the recovery of the unknown source. The numerical experiments carried out herein were implemented using the FEM and validated the numerical procedure. The analysis 


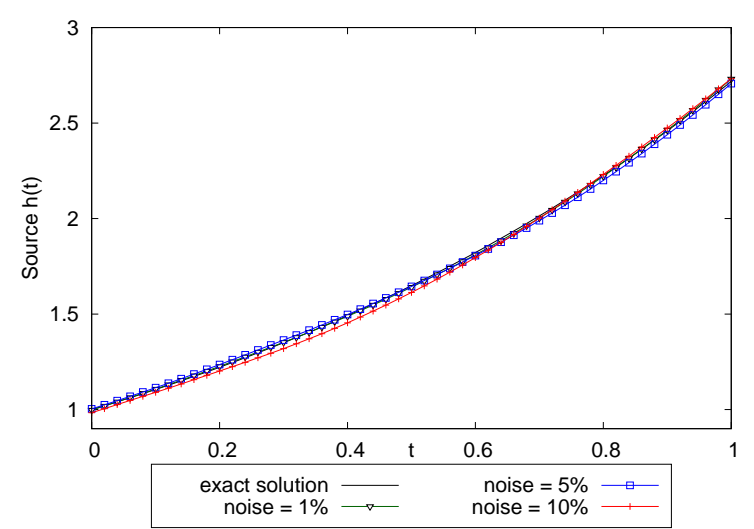

(a)

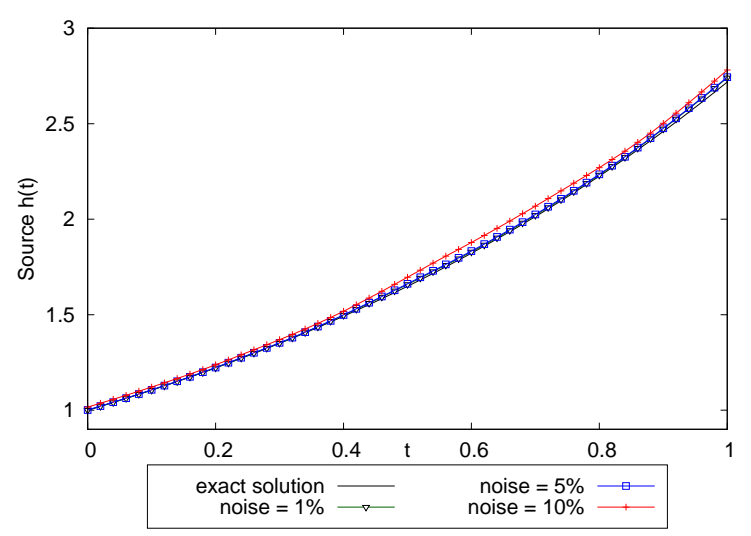

(c)

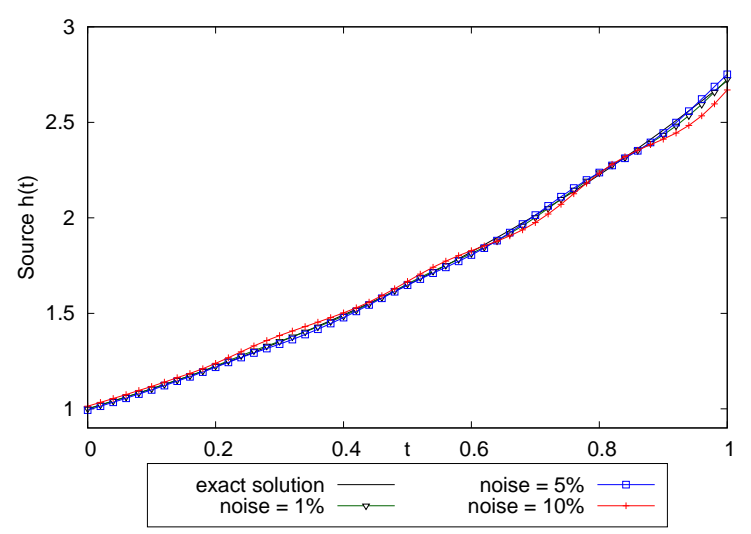

(e)
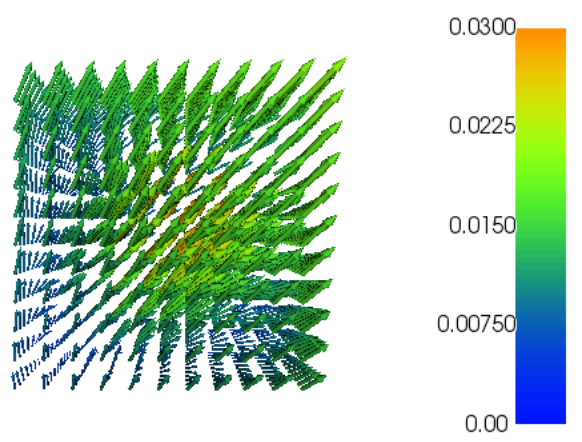

(b)

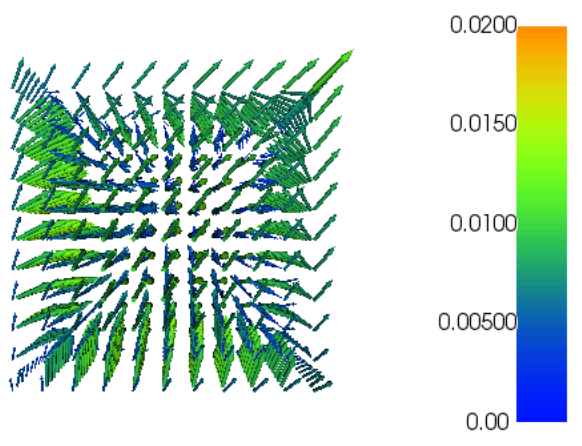

(d)

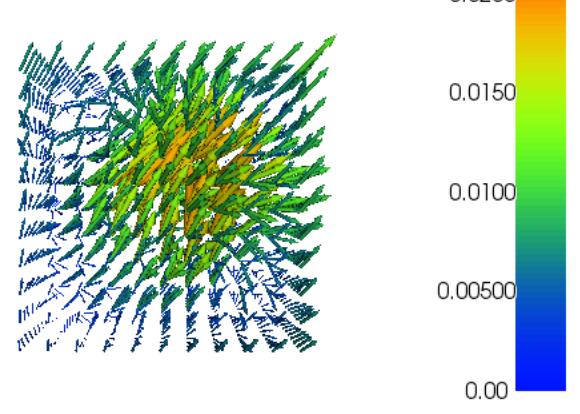

(f)

Figure 1. The exact source $h(t)=\exp (t)$ and its numerical approximations in Experiment 1, 2 and 3 using various levels of noise (a,c,e) and the error $\boldsymbol{E}-\boldsymbol{E}_{\mathrm{ex}}$ (and its absolute value) at final time $T=1$ for each experiment when $\tilde{e}=5 \%$ (b,d,f). Only 50 time points are used to plot the numerical approximations. 
Table 1. The CPU time (in minutes), the error $e(\boldsymbol{A})=\sum_{i=1}^{n}\left\|\boldsymbol{A}_{i}-\boldsymbol{A}_{\mathrm{ex}}\left(t_{i}\right)\right\|^{2} \tau$, the error $e(\phi)=\sum_{i=1}^{n}\left\|\phi_{i}-\phi_{\mathrm{ex}}\left(t_{i}\right)\right\|^{2} \tau$, the error $e(\boldsymbol{E})=\sum_{i=1}^{n}\left\|\boldsymbol{E}_{i}-\boldsymbol{E}_{\mathrm{ex}}\left(t_{i}\right)\right\|^{2} \tau$, and the error $e(h)=\max _{1 \leq i \leq n}\left|h_{i}-h_{\mathrm{ex}}\left(t_{i}\right)\right|$ obtained for the different numerical experiments (without noise).

\begin{tabular}{l|ccccc}
\hline & CPU time & $e(\boldsymbol{A})$ & $e(\phi)$ & $e(\boldsymbol{E})$ & $e(h)$ \\
\hline Experiment 1 & 91 & $1.162665 \mathrm{e}-06$ & $3.720334 \mathrm{e}-08$ & $1.038982 \mathrm{e}-04$ & $1.211658 \mathrm{e}-02$ \\
Experiment 2 & 182 & $5.734231 \mathrm{e}-07$ & $1.812047 \mathrm{e}-08$ & $1.271689 \mathrm{e}-05$ & $1.649774 \mathrm{e}-02$ \\
Experiment 3 & 192 & $3.000904 \mathrm{e}-08$ & $9.573042 \mathrm{e}-09$ & $2.687462 \mathrm{e}-05$ & $2.184774 \mathrm{e}-02$ \\
\hline
\end{tabular}

Table 2. The error $e(h)=\max _{1 \leq i \leq n}\left|h_{i}-h_{\mathrm{ex}}\left(t_{i}\right)\right|$ obtained for the different numerical experiments for various noise levels.

\begin{tabular}{c|ccc}
\hline$e(h)$ & $\tilde{e}=1 \%$ & $\tilde{e}=5 \%$ & $\tilde{e}=10 \%$ \\
\hline Experiment 1 & $2.263878 \mathrm{e}-05$ & $2.669173 \mathrm{e}-02$ & $3.786704 \mathrm{e}-02$ \\
Experiment 2 & $2.173975 \mathrm{e}-02$ & $2.540045 \mathrm{e}-02$ & $6.289425 \mathrm{e}-02$ \\
Experiment 3 & $2.779261 \mathrm{e}-02$ & $3.292474 \mathrm{e}-02$ & $7.828984 \mathrm{e}-02$ \\
\hline
\end{tabular}

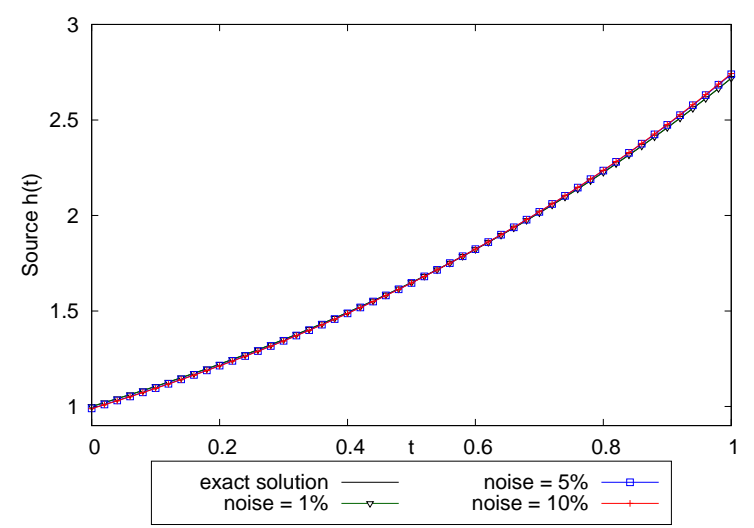

(a)

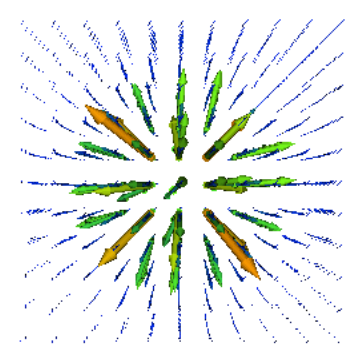

(c)
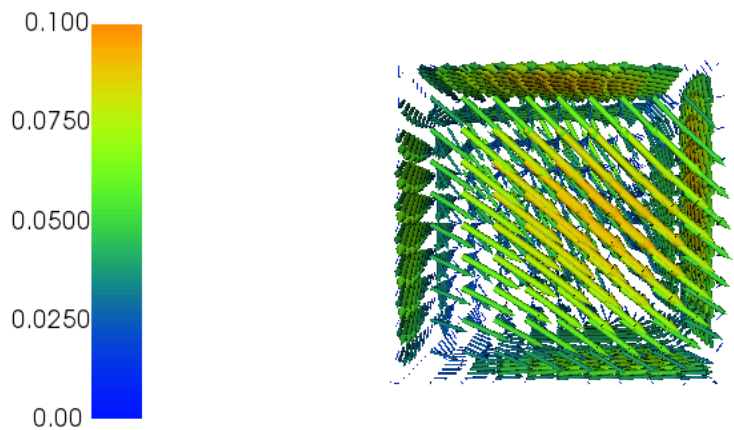

(d)

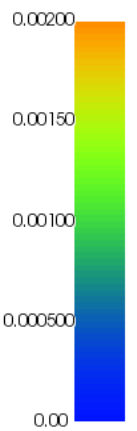

(b)

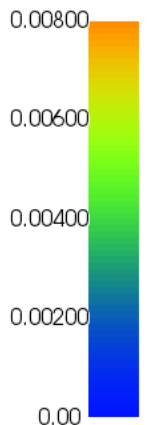

Figure 2. The exact source $h(t)=\exp (t)$ and its numerical approximations in Experiment 4 using various levels of noise (a) and the error $\boldsymbol{A}-\boldsymbol{A}$ ex, $\boldsymbol{E}-\boldsymbol{E}_{\mathrm{ex}}$ and $\boldsymbol{B}-\boldsymbol{B}_{\mathrm{ex}}$ (and its absolute value) at final time $T=1$ for the experiment when $\tilde{e}=5 \%$ (b,c,d). Only 50 time points are used to plot the numerical approximations. 
stays valid in two dimensions. Future work can be concerned with the derivation of the error estimates for the time and space discretization and with the improvement of the nodal FEM used in this paper.

Acknowledgements. This research is supported by National Basic Research Program of China under grant number 2014CB845906 and National Science Foundation of China (Grant Nos. 41590864, 41274103, 11571352). It is also partially supported by the Strategic Priority Research Program (B) of the Chinese Academy of Sciences (XDB 18010202). 


\section{References}

[1] O. Bíró, K. Preis, On the use of the magnetic vector potential in the finite element analysis of three-dimensional eddy currents, IEEE Tran. Magn. 25(1989), 3145-3159.

[2] A. Erdem, D. Lesnic, A. Hasanov, Identification of a spacewise dependent heat source, Appl. Math. Model., 37(2013), 10231-10244.

[3] W. C. Chew, Vector Potential Electromagnetic Theory with Generalized Gauge for Inhomogeneous Anisotropic Media, arXiv:1406.4780v1 [physics. class-ph], 2014.

[4] V. Girault, Incompressible finite element methods for Navier-Stokes equations with nonstandard boundary conditions in $R^{3}$, Math. Comput., 51(1988), 183, 55-74.

[5] S. Li, An Inverse Problem for Maxwell's Equations in Bi-isotropic Media, Siam J. Math. Anal., 37(2005), 1027-1043.

[6] S. Li, M. Yamamoto, An inverse problem for Maxwell's equations in isotropic and non-stationary media, Applicable Analysis, 92(2013), 23352356.

[7] T. Kang, K. I. Kim, Fully discrete potential-based finite element methods for a transient eddy current problem, Computing, 85(2009), 339-362.

[8] T. Kang, T. Chen, H. Zhang and K. I. Kim, Fully discrete $\boldsymbol{A}-\boldsymbol{\phi}$ finite element method for Maxwell's equations with nonlinear conductivity, Numer. Meth. Part. D. E., 30(2014), 2083-2108.

[9] A. Hasanov, Simultaneous determination of source terms in a linear parabolic problem from the final overdetermination: Weak solution approach, J. Math. Anal. Appl., 330(2007), 766-779.

10] M. Ismailov, F. Kanca, D. Lesnic, Determination of a time-dependent heat source under nonlocal boundary and integral overdetermination conditions, Appl. Math. Comput., 218(2011), 4138-4146.

[11] T. Johansson, D. Lesnic, A variational method for identifying a spacewise dependent heat source, IMA J. Appl. Math., 72(2007), 748-760.

[12] J. Kačur, Method of Rothe in Evolution Equations, in: Teubner Texte zur Mathematik, vol. 80, Teubner, Leipzig, 1985.

[13] T. Kang, T. Chen, H. Zhang, K. I. Kim, $\boldsymbol{A}$ - $\boldsymbol{\phi}$ finite element method with composite grids for time-dependent eddy current problem, Appl. Math. Comp., 267(2015), 365-381.

[14] V. Melicher, M. Slodička, Determination of missing boundary data for a steady-state Maxwell problem, Inverse Problem, 22(2006), 297-310.

[15] V. Melicher, M. Slodička, Boundary data identification for a eddy-current problem on polyhedra: numerical approach, J. Inverse Ill-Posed Problem, 14:3(2006), 279-293.

[16] A. Prilepko, D. Orlovsky, I. Vasin, Methods for Solving Inverse Problems in Mathematical Physics, in: Monographs and Textbooks in Pure and Applied Mathematics, vol. 222, Marcel Dekker, Inc., New York, Basel, 2000.

[17] M. Slodička, Determination of a solely time-dependent source in a semilinear parabolic problem by means of boundary measurements, J. Comput. Appl. Math., 289(2015), 433-440.

[18] M. Slodička, M. Galba, Recovery of a time dependent source from a surface measurement in Maxwell's equations, Comput. Math. Appl., 71(2015), 368-380.

[19] V.V. Solov'ev, Solvability of the inverse problems of finding a source, using overdetermination on the upper base for a parabolic equation, Differential Equations, 25(1990), 1114-1119.

20] K. Van Bockstal, R. H. De Staelen, M. Slodička, Identification of a memory kernel in a semilinear integrodifferential parabolic problem with applications in heat conduction with memory, J. Comput. Appl. Math., 2015, 289, 196-207.

[21] M. Yamamoto, On an inverse problem of determining source terms in Maxwell's equations with a single measurement, Inverse Problems Tomography \& Image Processing, Springer US, 1998, 241-256.

[22] Z. Zeng, L. Udpa, S. S. Udpa, M. S. Chan, Reduced magnetic vector potential formulation in the finite element analysis of eddy current nondestructive testing, IEEE Trans. Magn., 45(2009), 964-967.

[23] O.C. Zienkiewicz, Finite element - the basic concepts and the application to 3D magnetostatic problems, John Wiley \& Sons, INC., London, 1980.

[24] K. Van Bockstal, M. Slodička, Recovery of a space-dependent vector source in thermoelastic systems, Inverse Probl. Sci. Eng., 23 (6) (2015), 956-968.

[25] L. C. Evans, Partial differential equations, volume 19 of Graduate Studiein Mathematics, Providence, RI: American Mathematical Society, USA, 1998.

[26] J. Kačur. Method of Rothe in evolution equations, volume 80 of Teubner Texte zur Mathematik, Teubner, Leipzig, 1985.

[27] K. Van Bockstal, M. Slodička, Recovery of a time-dependent heat source in one-dimensional thermoelasticity of type-III, Inverse Problems Sci. Eng. (accepted), 2016.

[28] A. Logg and G. N. Wells, DOLFIN: Automated Finite Element Computing, ACM Trans. Math. Software, 37(2):28, 2010.

[29] A. Logg, G. N. Wells and J. Hake, DOLFIN: a C++/Python Finite Element Library, in Automated Solution of Differential Equations by the Finite Element Method, Volume 84 of Lecture Notes in Computational Science and Engineering, Edited by A. Logg, K.-A. Mardal and G. N. Wells, Springer, chapter 10, 2012.

[30] A. Logg, K.-A. Mardal, G. N. Wells et al., Automated Solution of Differential Equations by the Finite Element Method, Springer, 2012.

[31] M. S. Alnaes, J. Blechta, J. Hake, A. Johansson, B. Kehlet, A. Logg, C. Richardson, J. Ring, M. E. Rognes and G. N. Wells, The FEniCS Project Version 1.5, Archive of Numerical Software, vol. 3, 2015.

[32] M. Costabel and M. Dauge, Weighted regularization of Maxwell equations in polyhedral domains, Numerische Mathematik, vol. 93:2(2002), 239-277.

[33] J.-M. Jin. The Finite Element Method in Electromagnetics. Wiley-IEEE Press, 3rd edition, 2014. 